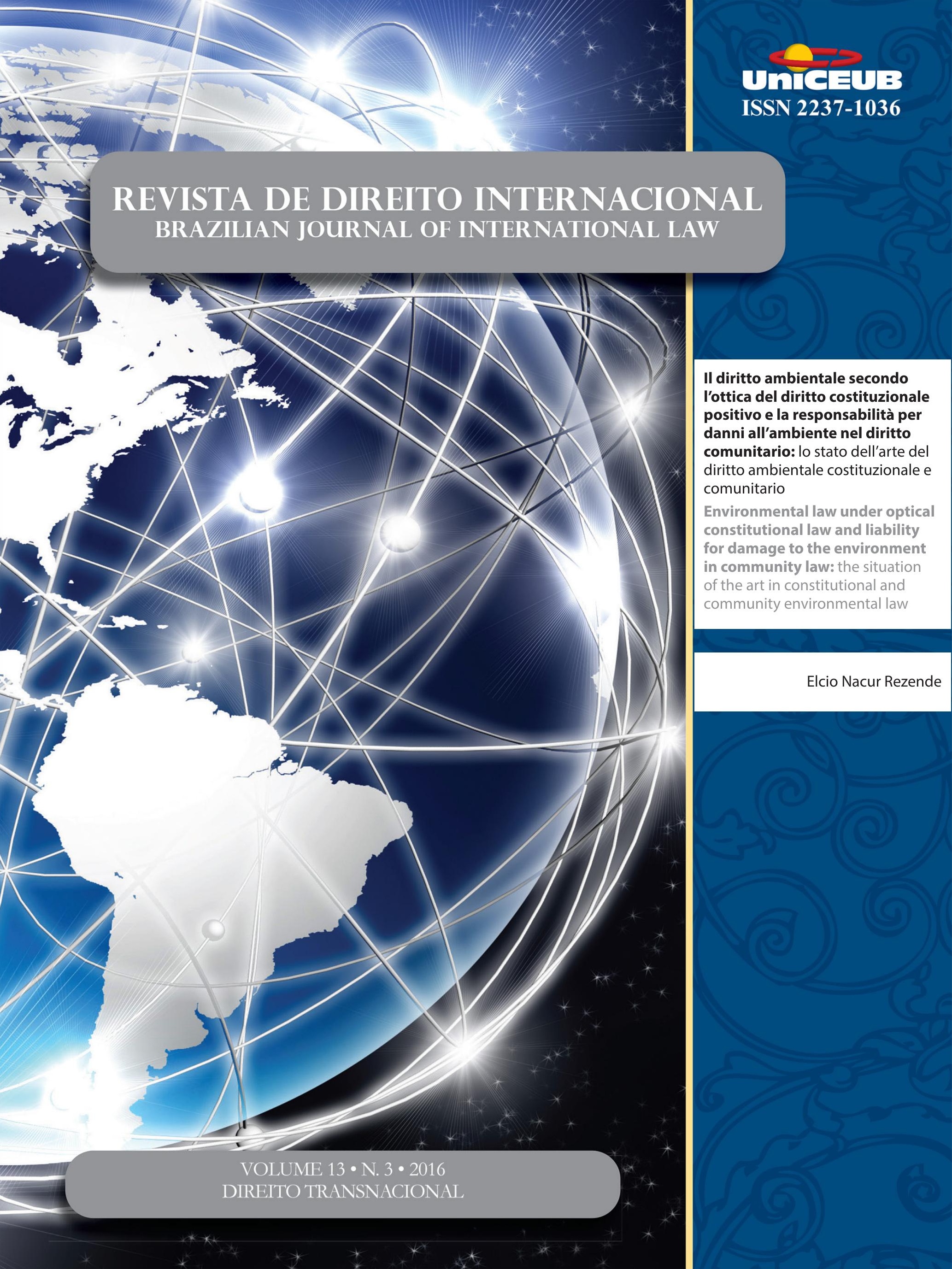


Crônicas da ATUALIdAde do direito internacional .................................................. 2

I. Dossiê Temático: Direito Transnacional .........................................................15

EDITORIAL: O Direito Transnacional - Circulação de normas e relações jurídicas transnacionais .......16 Priscila Pereira de Andrade

A emergênCia do direito transnacional ambiental .............................................18 Priscila Pereira de Andrade

Desafíos y RESPUESTAS TRANSNACIONALES FRENTE A LOS CRÍMENES AMBIENTALES ...............30 Rosmerlin Estupiñan-Silva

DiREITO TRANSNACIONAL E MUdANÇAS CLIMÁTICAS .50 Géraud de Lassus Saint-Geniès

Especies en movimiento: la Convención sobre el Comercio Internacional de Especies Amenazadas de Fauna y Flora Silvestres como espacio de “Encuentro” de discursos, ACTORES Y ESTRATEGIAS EN EL DERECHO AMBIENTAL TRASNACIONAL

María Valeria Berros e Dabel Leandro Franco

El carácter transnacional del Sistema comunitario de ECogestion « Eco-ManageMENT AND Audit SCHEME » (EMAS) DENTRo de LA UE y MÁs ALlÁ DE SUS Fronteras ......72 Adélie Pomade

O CONCEITO DE CONDUTA EMPRESARIAL RESPONSÁVEL À LUZ DOS ORDENAMENTOS JURÍDICOS BRASILEIRO, INTERNACIONAL E TRANSNACIONAL

Gabriel Webber Ziero

ARBITRAGEM NO DIREITO TRIBUTÁRIO INTERNACIONAL E NO DIREITO INTERNACIONAL DOS INVESTIMENTOS: UMA MANIFESTAÇÃO DO DIREITO TRANSNACIONAL

Vivian Daniele Rocha Gabriel 
O DIREITO TRIBUTÁRIO SOB UMA PERSPECTIVA TRANSNACIONAL

Franciele de Simas Estrela Borges

As Características do Direito Transnacional como Metodologia: Análise sob o enfoQue dos Aspectos Processuais da Arbitragem 126

Flávia Foz Mange

O DIREITO TRANSNACIONAL (“GLOBAL LAW") E A CRISE DE PARADIGMA DO ESTADO-CENTRISMO: É POSSÍVEL CONCEBER UMA ORDEM JURÍDICA TRANSNACIONAL? ...................................... 146

Luiza Nogueira Barbosa e Valesca Raizer Borges Moschen

TransPorte AÉREO E DIREITO TRANSNACIONAL: DA CONVERGÊNCIA À UNIFORMIDADE 160 Mickael R. Viglino

Outros Artigos. 175

O Fundo Monetário Internacional e a proteção dos direitos humanos: uma análise DO PROGRAMA DE CRESCIMENTO E REDUÇÃO DA POBREZA NO HAITI 177

Pablo Henrique Hubner de Lanna Costa e Carlos Alberto Simões de Tomaz

Um estranho no ninho? Padrões privados no Acordo de Barreiras Técnicas ao CoMÉRCIO DA OMC 192

Michelle Ratton Sanchez Badin e Marina Yoshimi Takitani

Os benefícios tributários do programa Inovar-Auto e os princípios da Nação Mais Favorecida e do Tratamento Nacional: uma análise dos argumentos dos Painéis atualmente em Curso contra o Brasil no Órgão de SoluÇão de Controvérsias da OMC . 211 Eric Moraes Castro e Silva

A ERA DA HUMANIDADE: REFLEXões PARA A HISTÓRIA DO DIREITO INTERNACIONAL 236 Henrique Weil Afonso

Precedentes vinculantes nos Estados Unidos da América e no direito brasileiro: Um ESTUDO COMPARADO 264

Patrícia Perrone Campos Mello 
IL DIRITTO AMBIENTALE SECONDO L'OTTICA DEL DIRITTO COSTITUZIONALE POSITIVO E LA RESPONSABILITÀ PER DANNI ALL'AMBIENTE NEL DIRITTO COMUNITARIO: LO STATO DELL'ARTE DEL DIRITTO AMBIENTALE COSTITUZIONALE E COMUNITARIO 287

Elcio Nacur Rezende

DA DESCONSIDERAÇÃo DA PERSONALIDADE JURÍDICA NAS RELAÇÕES CONSUMEIRISTAS BRASILEIRAS: ANÁLISE À LUZ DAS TEORIAS CLÁSSICAS

Daniel Amin Ferraz e Marcus Vinicius Silveira de Sá

ANALYSIS OF ADVANTAGES AND DISADVANTAGES OF FORUMS PRESCRIBED UNDER THE UNCLOS AND STATE PRACTICE: THE WAY AHEAD FOR INDIA ......................................................319

Vinai Kumar Singh

Do governo POR LEIS À governanÇA POR NúMERos: breve anÁlise do Trade in SERVICE AgreEMENT (TISA) ...............................................................................338 Jânia Maria Lopes Saldanha, Rafaela da Cruz Mello e Têmis Limberger

As DIRETIVAS EUROPEIAS COMO NORMA REGULADORA DO DIREITO ADMINISTRATIVO GLOBAL ..356 Alice Rocha da Silva e Ruth Maria Pereira dos Santos

O desenVolvimento da POlítica AGRícola COMUM dA UNião EUROPEIA 375 Tatiana de A. F. R. Cardoso Squeff

A imunidade de Jurisdição das organizaÇões internacionais FaCE AO Direito de aCESSO À JUSTIÇA 391

Fernanda Araújo Kallás e Caetano

O DIREITO INTERNACIONAL ENTRE O DEVER ÉTICO E A AÇÃo POLÍ́TICA: OS FUNDAMENTOS DE UM DEVER DE COOPERAÇÃO INTERNACIONAL NA FILOSOFIA POLÍTICA DE IMMANUEL KANT .405 Ademar Junior Pozzatti

EXTENSÃo E FRAGMENTAÇÃo NO CONTEXTO DA JURISDIÇÃO PENAL INTERNACIONAL .423 Marcus Vinícius Xavier de Oliveira

A DEFINiÇÃo JURÍdiCA DA "COMUNIDADE" .444 Nitish Monebhurrun, Michelle Lucas Cardoso Balbino, Fernanda Castelo Branco Araujo, Othon Pantoja, Míara Bogo Bruno e Cândida Dettenborn Nóbrega 
Comparative Study on Chinese Local Legislation of Science and Technology ProGRESS

LI Xiaoming e LI Yihan

O CONTROLE PENAL DO TRÁFICO DE PESSOAS: CONSTRUÇÃO JURÍDICA, INTERAÇÕES ORGANIZACIONAIS E COOPERAÇÃO INTERNACIONAL

Bruno Amaral Machado e Priscilla Brito Silva Vieira

Desativismo judicial: a extradição Battisti no Supremo Tribunal Federal .505 Francisco Rezek e Israel Paulino

A decisão norte-americana do Caso Myriad: novos paradigmas para a Proteção patenTÁRIA DO CÓDIGO GENÉTICO HUMANO E BIOTECNOLOGIA 514 José Carlos Vaz e Dias e Clarisse De La Cerda 


\section{Il diritto ambientale secondo l'ottica del diritto costituzionale positivo e la responsabilità per danni all'ambiente nel diritto comunitario: lo stato dell'arte del diritto ambientale costituzionale e comunitario*}

\section{Environmental law under optical constitutional law and liability for damage to the environment in community law: the situation of the art in constitutional and community environmental law}

Elcio Nacur Rezende**

\section{SOMMARIO}

Inequivocabilmente, la tutela dell'ambiente è uno dei più grandi cura dell'essere umano in epoca contemporanea.

Per quanto riguarda la scienza giuridica, il testo di questo lavoro dimostra che la legge ambientale viene progressivamente in fase di studio da diversi giuristi di tutto il mondo, in particolare con l'intenzione di costruire un quadro giuridico in grado di sostenere che la cura per l'ambiente è l'obbligo dello Stato e di tutti i cittadini.

In tale ottica, l'ambiente ecologicamente equilibrato raggiunto in diversi sistemi giuridici del diritto fondamentale di status, dando origine al ruolo dei principi costituzionali in diversi testi di paesi di tutti i continenti.

Con corollario, in quanto è dedicata alla tutela dell'ambiente come diritto fondamentale, si impone degradare il dovere di responsabilità per danni ambientali.

Con questi presupposti, il documento presenta l'ultimo stato dell'arte per quanto riguarda la legge costituzionale ambientale positivizzato, presentando il testo delle costituzioni di molti paesi di tutti i continenti, e tuttavia, il diritto comunitario è oggetto di responsabilità ambientale nella sua direttiva attuale, che è favorevole alla giocatore ragionamento giuridico in grado di fornire con una riflessione sul tema che, come detto, ha un valore incommensurabile senza dubbio alla vita umana.

Parole: Diritto Ambientale; Diritto Costituzionale; Responsabilità Ambientale.
*** Pós-Doutor pela Universidade de Messina. Doutor e Mestre pela PUC-Minas. Professor do Programa de Pós-graduação em Direito da Escola Superior Dom Helder Câmara. Procurador da Fazenda Nacional. E-mail: elcionrezende@yahoo.com.br
Recebido em 13/07/2016

** Mestrando em Proteção dos Direitos Fundamentais (Universidade de Itaúna). Advogado. E-mail: ca.tomaz@uol.com.br 


\section{Abstract}

Undoubtedly, environmental preservation is one of the greatest care of the Human Being in the contemporary world.

With regard to Juridical Science, the text of this work demonstrates that Environmental Law has been progressively studied by several jurists all over the world, mainly with the intention of building a legal framework capable of justifying that care for the environment which is the obligation of the State and of all citizens.

Within this framework, the ecologically balanced Environment has achieved in several legal systems the status of Fundamental Law, becoming part of the Constitutional Principles in various texts from countries of all continents.

As a logical corollary, insofar as environmental preservation is enshrined as a Fundamental Right, destroyers must be held accountable for damages to the environment.

With these premises, the work presents the most recent State of the Art concerning Positive Environmental Constitutional Law, presenting the constitutional text of several countries of all continents and, nevertheless, how Law Community Law deals with the theme of Environmental Responsibility in its Directive in order to offer to the reader a legal basis capable to provide a reflection about the subject which, as said, has undoubtedly an immeasurable value for human life

Keywords: Environmental Law; Constitutional law; Environmental Liability.

\section{INTRODUZIONE}

La preservazione dell'Ambiente ha raggiunto, nei nostri tempi, un valore giuridico massimo, integrando addirittura, nel Diritto Costituzionale di vari Paesi, la categoria di Diritto Fondamentale.

In effetti, la preservazione ambientale è intimamente legata alla Dignità della Persona Umana, poiché, senza uno sforzo intellettuale, si deduce facilmente che è impossibile che l'Essere Umano possieda una qualità di vita ragionevole se non s'inserisce in un ambiente sano.

Tuttavia, è inesorabile che, lamentabilmente, il mon- do soffra grandi tragedie ambientali che cagionano, costantemente, la perdita della vita umana e il deterioramento dell'Ambiente il quale, molte volte, non riuscirà a recuperarsi.

Di fronte a questo quadro, il presente lavoro dimostrerà la necessità che, partendo dalla consacrazione del Diritto Fondamentale all'Ambiente Ecologicamente Equilibrato, previsto in molte Costituzioni, è imprescindibile, anche come corollario giuridico ovvio, che s'imputi Responsabilità a quelli che cagionano deterioramento all'Ambiente attraverso la consacrazione giuridica della tutela ambientale.

\section{LA CORRELAZIONE TRA IL DIRITTO COSTITUZIONALE E IL DIRITTO AMBIENTALE}

Il Diritto Costituzionale contemporaneo, attento ai beni giuridici che meritano tutela fondamentale, conferisce valore massimo all'Ambiente Ecologicamente Equilibrato, eleggendolo anche come uno dei beni della vita che meritano la massima attenzione tanto dallo Stato quanto dalla Società.

Partendo da questa premessa, i costituzionalisti trattano sempre di più il Diritto Ambientale come un ramo giuridico di estrema importanza, non dissociandolo, neanche riducendolo, dinanzi ad altri rami del Diritti più antichi quali il Diritto Penale, Civile, Amministrativo o Tributario. Al contrario, si costata che l'ambientalismo ha ricevuto nella concezione giuridica degli ultimi decenni un'attenzione particolare.

Il Diritto Costituzionale come ramo giuridico che concentra il suo studio sui beni della vita di grande valorizzazione giuridica, attento alla necessità imperiosa della protezione dell'Ambiente come fattore imprescindibile al mantenimento salutare della vita umana, ha innalzato lo studio del Diritto Ambientale al livello massimo, incorporando dei principi tipici dell'ambientalismo all'elenco della "principiologia" costituzionale.

In questo senso, svolgendo un'analisi delle questioni ambientali da una prospettiva costituzionalista italiana, insegna Antonio Gusmai ${ }^{1}$ :

1 GUSMAI, Antonio. La tutela costituzionale dell'ambiente tra valori (meta-positivi), interessi (mercificatori) e (assenza di) principi fondamentali. C.f. $<$ http://www.edizioniesi.it/dperonline/data/uploads/articoli/tutela-gusmai.pdf>. Accesso in: 27 apr. 2016. 
Volendo provare a tirare le somme in merito a quanto sinora detto, sembra innanzitutto potersi rilevare, se non ci s'inganna, una certa "debolezza" e complessiva frammentarietà dell'odierna tutela costituzionale del bene giuridico ambiente.

La necessità, evidenziata poco sopra, di promuovere a principio costituzionale espresso il bene giuridico ambiente (magari inserendolo, considerata la rilevanza, tra quelli c.d.intoccabil in quantofondamentale), ha, infatti, il principale scopo di allignare tale bene all'interno di una dimensione costituzionale - quella repubblicana in cui appare ancora possibile (perlomeno in parte) materialmente difenderlo dagli interessi predatori di un'economia globale sempre più rispondente alle distruttive logiche di un mercato concorrenziale libero e senza regole.

Il modello di democrazia sociale voluto dai Padri costituenti, ossia la realizzazione progressiva del programma di trasformazione economico-sociale previsto dall'art. 3, comma 2, Cost., sembra invero essere l'unico modello compatibile con uno sviluppo sostenibile di un'economia realisticamente attenta e sensibile alle problematiche ambientali.

Se è vero com'è vero che i diritti fondamentali si pongono a presidio della vita, che in nessuna sua manifestazione può essere attratta nel mondo delle merci, non può non essere allora ancor più vero che proprio l'ambiente, condizionando la materiale esistenza stessa della vita, necessiti di una tutela insuscettibile di essere pretermessa alle libertà d'iniziativa economico-privata.

Tuttavia, l'ermeneutica costituzionale è divenuta di estrema importanza nell'esegesi normativa del Diritto Penale Ambientale, del Diritto Tributario Ambientale, del Diritto Amministrativo Ambientale e, ovviamente, in materia di Responsabilità per Danno all'Ambiente.

$\mathrm{Si}$ registri, pertanto, che consentaneamente all'innalzamento della preservazione ambientale al livello di Diritto Fondamentale Costituzionale, è diventato necessario che l'imputazione al responsabile del degrado avesse anche un trattamento costituzionale. Si costata, dunque, che i costituzionalisti contemporanei, in maniera lodevole, nell'eleggere la preservazione ambientale come diritto fondamentale, hanno anche conferito, a quello che degrada l'Ambiente, una responsabilità implacabile, distinguendo l'illecito ambientale dagli altrettanti illeciti (civile, amministrativo o penale) dimostrando così che il danno ambientale possiede, sicuramente, particolarità che lo differenziano dagli altri.

Procedendo oltre al Diritto Costituzionale Classico, contemporaneamente, si sostiene che la preservazione ambientale dev'essere trattata come Diritto Fondamen- tale Umano, come spiega Flora Vollero²:

\section{IL DIRITTO AD UN AMBIENTE SALUBRE COME DIRITTO FONDAMENTALE}

Uomo, ambiente e " questione ambientale".

L'ambiente è il luogo in cui l'uomo vive e, inevitabilmente, nello svolgimento della sua esistenza egli non ha potuto ignorare lo spazio che lo circondava; non è esatto dire, dunque, che la problematica ambientale sia nata solo negli ultimi anni: la necessità di regolare il rapporto uomo- natura è sempre esistita, ciò che muta è l'atteggiamento dell'uomo nei confronti dell'ambiente, ossia il valore a esso attribuito.

Se, infatti, fino a un periodo poi non molto lontano, l'ambiente era visto, per lo più, come uno spazio più o meno liberamente appropriabile secondo quelle che erano le generali regole disciplinanti i rapporti intersoggettivi, negli ultimi anni la rilevanza dell'ambiente assume connotati tutt'altro diversi, rappresentando esso stesso un valore.

In effetti, da una nozione antropocentrica, e, si potrebbe dire, proprio per questo utilitaristica dell'ambiente (esso esiste in funzione dell'uomo e trova nell'uomo a un tempo la sua ragione ed il suo limite), si passa ad una serie di concetti e di valori che non vedono nell'esprit de conquête da parte dell'uomo il valore in sé, che trova un limite nell'utilità complessiva di questa sorta di "signore", ma considera come prius l'ambiente stesso.

Assistiamo, dunque, a un vero mutamento di prospettiva in cui quel rapporto viene articolato in maniera più complessa.

La crescente aggressione all'ambiente, incidendo in maniera sempre più vistosa sulla qualità della vita, ha sicuramente favorito una riconsiderazione delle visione meramente "utilitaristica", ma le basi concettuali di questa rivisitazione si ritrovano anche nei valori che hanno ispirato la nostra come altre costituzioni contemporanee: in particolare, nella dimensione personalista che ne costituisce il principio guida fondamentale

Al livello del Diritto Costituzionale Positivo è percettibile la consacrazione della preservazione ambientale come valore meritevole di tutela nella misura in cui le costituzioni di molte nazioni eleggono nei loro testi l'Ambiente come Diritto Fondamentale.

Secondo studi svolti dalla Fundação David Suzuki ${ }^{3}$, più di 140 Paesi hanno alterato la loro Costituzione

2 VOLLERO, Flora. Diritti umani e diritti fondamentali fra tutela costituzionale e tutela sovranazionale:il diritto ad un ambiente salubre. C.f. $<$ http://files.studiperlapace.it/spp_zfiles/ docs/20041205175248.pdf>. Accesso in: 27 apr. 2016.

3 DAVID SUZUKI FUNDATION. C.f. $<$ http://www.davidsuzuki.org/ >.Accesso in: 09 mag. 2016. 
Federale per esigere protezione ambientale, e 98 Paesi hanno riconosciuto espressamente il diritto costituzionale di vivere in un ambiente sano.

\subsection{L'Ambiente nelle Costituzioni Europee}

Passiamo quindi a controllare se la premessa fatta è corroborata dal Diritto Costituzionale Positivo europeo.

La Costituzione della Bulgaria ${ }^{4}$, datata del 1971, è stata una delle prime a determinare che è imposto allo Stato e alla Società il dovere della preservazione ambientale, elencando l'aria, il suolo e l'acqua come beni d'inestimabile valore.

In Germania ${ }^{5}$, l'emendamento del 1994, ha disposto che è dovere statale garantire alle future generazioni le risorse naturali, imponendo ai Poteri dello Stato attuare nella produzione di norme, nell'amministrazione e nella prestazione giurisdizionale in modo a garantire un Ambiente sano per il futuro.

La Costituzione del Belgio ${ }^{6}$, seguendo gli stessi parametri, elegge anche l'Ambiente come bene da essere tutelato da tutti.

In Svizzera ${ }^{7}$, oltre alla semplice preservazione ambientale, si è anche risaltato, nel suo articolo 24 , che la manipolazione genetica deve rispettare la Dignità della creazione animale e vegetale. Nel suo testo del 1999, la Carta impone protezione all'Ambiente naturale a scapito degli interessi individuali, oltre a evidenziare il Principio della Precauzione, imponendo comportamenti negativi che, eventualmente possano degradare l'Ambiente. Si rilevi che l'ambiente è protetto anche da varie leggi infra-costituzionali denominate Bundesgeset\%, o "Acts", essendone la principale, la Legge Federale di Protezione all'Ambiente del 1983, conosciuta anche come LPA, Legge di Protezione Ambientale ${ }^{8}$

4 COSTITUZIONE DELlA BULGARIA. C.f.<http://www. egov.ufsc.br/portal/sites/default/files/anexos/21362-21363-1-PB. htm >. Accesso in 09 mag. 2016.

5 LEGGE FONDAMENTALE DELLA REPUBBLICA FEDERALE DI GERMANIA.C.f.<https://www.btg-bestellservice.de/ pdf/80208000.pdf> . Accesso in: 20 apr. 2016.

6 BELGIUM CONSTITUTION. C.f.<http://www.egov.ufsc. br/portal/sites/default/files/anexos/21393-21394-1-PB.htm> Accesso in: 20 apr. 2016.

7 CONFEDARAZIONE SVIZZERA. C.f. $<$ http://www.admin. ch>. Accesso in: 20 apr. 2016.

8 CONFEDARAZIONE SVIZZERA.: Umweltschutzgeset\%. O USG. Traduzione inglese: EPA (Environmental Protection Act). (Legge di Protezione Ambientale Svizzera).
La Costituzione della Francia9 esalta la valorizzazione ambientale in vari dispositivi, a cominciare dal preambolo quando dispone: "Il popolo francese proclama solennemente il suo compromesso riguardo ai diritti umani e i principi della sovranità nazionale, come definito dalla Dichiarazione del 1789, confermata e completata dal Preambolo della Costituzione del 1946, così come dai diritti e doveri definiti nella Carta Ambientale del 2004.” Oltre al preambolo, nel 2004 è stata promulgata la Carta Ambientale che dispone:

\section{Il testo della Carta per l'Ambiente}

Il popolo francese considerando, che le risorse e gli equilibri naturali hanno condizionato l'emergenza dell'umanità; che il futuro e l'esistenza stessa dell'umanità sono indissociabili dall'ambiente naturale; che l'ambiente è il patrimonio comune a tutti gli esseri umani; che l'uomo esercita una pressione crescente sulle condizioni di vita e sulla propria evoluzione; che la diversità biologica, il benessere delle persone e il progresso delle società umane sono affette da certi modi di consumo o di produzione e dall'eccessivo sfruttamento delle risorse naturali; che la protezione dell'ambiente deve essere messa allo stesso piano degli altri interessi fondamentali della Nazione; che per poter assicurare uno sviluppo durevole, le scelte il cui scopo è rispondere ai bisogni di oggi, non devono compromettere la capacità delle generazioni future e di altri popoli di soddisfare i propri bisogni;

proclama:

Art.1- Ognuno ha il diritto di vivere in un ambiente equilibrato e favorevole alla propria salute.

Art.2- Ogni persona ha il dovere di partecipare alla preservazione e al miglioramento dell'ambiente.

Art.3- Ogni persona deve, nelle condizioni stabilite dalla legge, prevenire o, a difetto, limitare i danni che sarebbe suscettibile fare all'ambiente.

Art.4- Ogni persona deve contribuire alla riparazione dei danni che causa all'ambiente, nelle condizioni stabilite dalla legge.

Art.5- Se la realizzazione di un danno, anche se incerto da un punto di vista scientifico, può influire in modo grave o irreversibile l'ambiente, le autorità politiche vigilano, per applicazione del principio di precauzione, sull'applicazione di misure provvisorie e proporzionate per poter evitare la realizzazione del danno e mettere in opera una procedura di evacuazione dei rischi incorsi.

Art.6- Le politiche pubbliche devono promuovere uno sviluppo durevole. Per questo, devono

9 CONSEIL CONSTITUTIONNEL FRANCE. C.f. $<$ http:// www.conseilconstitutionnel.fr/conseilconstitutionnel/root/bank_ $\mathrm{mm} /$ portugais/constitution_por tugais.pdf $>$. Accesso in: 21 apr. 2016. 
tener conto della protezione e la valorizzazione dell'ambiente e conciliarle con lo sviluppo economico e sociale.

Art.7- Ogni persona ha il diritto, nelle condizioni stabilite dalla legge, di avere accesso alle informazioni relative all'ambiente detenute dalle autorità pubbliche e di partecipare all'elaborazione di decisioni pubbliche avendo un'influenza sull'ambiente.

Art.8- L'educazione e le formazioni sull'ambiente devono contribuire all'applicazione dei diritti e doveri definiti nella presente Carta.

Art.9- La ricerca e le innovazioni devono aiutare alla preservazione e alla valorizzazione dell'ambiente.

Art.10- La presente Carta ispira l'azione europea e internazionale della Francia.

La Costituzione Austriaca ${ }^{10}$ datata del 1920, nei suoi articoli 10 e 11, fissa le competenze legislative ed esecutive in materia ambientale, attribuendo all'Unione (Bundesstaat) e agli Stati (Länder), rispettivamente, l'attribuzione concorrente, stabilendo che la protezione ambientale è di tutti gli enti federativi.

In Inghilterra, a causa della sua Costituzione sostenuta dalla Common Law, pertanto con carattere di diritto consuetudinario, non esiste norma protettiva ambientale positivata. Esistono, però, due tradizionali fonti di leggi: Statuti - Statute Law, e le Leggi Consuetudinarie - Common Law. Gli statuti sono originati dal parlamento, come, per esempio l'Environmental Protection Act, del 1990, che tratta i danni causati dal deposito di residui non autorizzati e il Water Resorces Act, 1991, che proibisce lo scarico di sostanze inquinanti nelle acque. Le leggi consuetudinarie, invece, sono derivate dalla giurisprudenza - Case Law. Così, si valorizzano i precedenti giudiziali quando si giudicano i danni ambientali.

La Costituzione della Norvegia ${ }^{11}$ è datata del 1814 e, pertanto, non fa riferimento alla protezione ambientale, poiché, come sappiamo, in quella data, purtroppo, $i$ testi costituzionali non avevano ancora valorizzato l'Ambiente. Tuttavia, l'imposizione del rispetto alla questione ambientale è sorta con il Pollution act, che è entrato in vigore nel 1989, disponendo che qualunque atto come: 1- Introdurre solidi, liquidi o gas in aria, acqua e suolo; 2- Suono o vibrazioni; 3- Luce o altra radia-

10 BUNDESKANZLERAMT RECHTSINFORMATIONSSYSTEM. C.f.<http://www.ris.bka.gv.at>. Accesso in: 20 apr. 2016. 11 NORVEGIA COSTITUZIONE. C.f.<https://pt.glosbe. $\mathrm{com} / \mathrm{pt} / \mathrm{en} /$ Constitui $\% \mathrm{C} 3 \% \mathrm{~A} 7 \% \mathrm{C} 3 \% \mathrm{~A} 3 \mathrm{o} \% 20 \mathrm{da} \% 20$ Noruega $>$. Acesso in: 13 apr. 2016. zione nell'estensione determinata dall'autorità di controllo d'inquinamento; 4- Effetti sulla temperatura che cagionano o possano dare origine a danno o infortunio all'Ambiente; è considerato inquinamento e, pertanto, passibile di responsabilizzazione.

La Costituzione della Spagna ${ }^{12}$ (1978) nel suo art. 45 difende la protezione dell'Ambiente nell'affermare che:

Art. 45. - 1) Tutti hanno diritto a fruire di un ambiente adeguato per lo sviluppo della persona, nonché il dovere di mantenerlo.

2) I poteri pubblici veglieranno sulla razionale utilizzazione di tutte le risorse naturali, al fine di proteggere e migliorare la qualità della vita, nonché di difendere e ripristinare l'ambiente, contando sull'indispensabile solidarietà collettiva.

3) A carico di quanti violino il disposto del comma precedente, nei termini fissati dalla legge, saranno previste sanzioni penali o, nel caso, amministrative, come pure l'obbligo di riparare al danno causato.

$\mathrm{Si}$ osserva che la protezione dell'Ambiente nella Costituzione Spagnola sostiene una responsabilità triplice nello stabilire sanzioni penali, civili e amministrative a chi causa un danno ambientale.

Per quanto riguarda la Costituzione Italiana ${ }^{13}$, è degna di nota la lezione di Claudio Pollio ${ }^{14}$ :

La Costituzione Repubblicana italiana, com'è noto, non affronta direttamente il tema della disciplina delle risorse naturali e non riconosce specificamente il diritto all'ambiente, infatti, in tutto il testo non compare mai la parola "ambiente".

Nelle Carte Costituzionali più recenti, sotto la spinta di una maturata coscienza ecologista, si è previsto il diritto all'ambiente, configurandolo come obbligo-dovere dello Stato, diretto alla preservazione e protezione delle risorse naturali, e ha avuto riconoscimento il diritto del singolo a vivere in un ambiente sano.

La mancanza di norme espresse e puntuali sull'ambiente nella Costituzione non impedisce di organizzare un modello basato su riferimenti costituzionali forniti dagli artt. 9, 32, 42, 44. L'art. 9 fa riferimento alla tutela del paesaggio da parte

12 RETE DI COOPERAZIONE GIURIDICA INTERNAZIONALE IBEROAMERICANA. Constituição Espanhola. C.f. $<$ https://www.iberred.org/pt/legislacion-penal/constituicaoespanhola-de-27-de-dezembro-de-1978>. Accesso in: 13 apr. 2016. 13 POLLIO, Claudio. Il senso diffuso di comunità constituisce la forza principale dell'italia. C.f.<http://www.quirinale.it/qrnw/statico/costituzione/pdf/Costituzione.pdf>. Acesso in: 20 apr. 2016. 14 POLLIO, Claudio. Il senso diffuso di comunità constituisce la forza principale dell'italia.. C.f.<http://www.puntacampanella.org/ public/documenti/tesi-aree-marine-protette-claudio-pollio.pdf $>$. Accesso in: 27 apr. 2016. 
della Repubblica, la dottrina, pur muovendo da interpretazioni diverse della suddetta locuzione, è generalmente concorde nel ritenere tale norma un importante principio costituzionale di tutela dell'ambiente. Il dibattito, sia dottrinario che giurisprudenziale si è svolto intorno all'ampiezza della nozione di paesaggio; un primo autorevole filone dottrinario, rifacendosi alla definizione di "bellezze naturali" contenuta nella legge 29 giugno 1939 n. 1497, considera la norma costituzionale come avente "di mira unicamente i valori paesistici sotto il profilo dei quadri naturali che essi realizzano" non ricadendo pertanto nella materia della disposizione in esame la natura in quanto tale.

$[\cdots]$

A conclusione, non è facile definire dove l'ambiente si collochi nella Costituzione, ma ciò che va sottolineato è il fatto che la politica ambientale investe una materia che è contigua a dei valori, cui il legislatore deve rispetto, data la loro rilevanza costituzionale.

Rafforza la questione adottata nel paragrafo precedente Daniele Gianinni ${ }^{15}$ :
La tutela dell'ambiente
L" "ambiente" costituisce un bene giuridico unitario di valore costituzionale primario.

L'evoluzione della giurisprudenza costituzionale ha contribuito al superamento della tradizionale tesi interpretativa che sosteneva la natura proteiforme della "materia ambiente", come tale capace di affasciare sia la tutela dei beni paesaggistici e culturali (ambiente culturale), sia la disciplina contro gli inquinamenti (ambiente ecologico), sia il governo del territorio (ambiente urbanistico).

E, infatti, col conforto di numerose pronunce della Corte Costituzionale, la dottrina e la giurisprudenza ritengono oramai pacificamente che la disciplina unitaria e complessiva del bene ambiente inerisca a un interesse pubblico di valore costituzionale "primario" e "assoluto", e deve garantire (come prescrive il diritto comunitario) un elevato livello di tutela, come tale inderogabile dalle altre discipline di settore.

Costituisce un dato di fatto, però, che la Costituzione non menzioni l'ambiente tra i principi fondamentali e i diritti dei cittadini.

Ciò nonostante, proprio in ragione del già annunciato rilievo primario e assoluto del "bene giuridico ambiente", l'interesse ambientale viene unanimemente qualificato come interesse di rilevo costituzionale, protetto in particolare dagli artt. 9, comma 2, e 32 Cost.

15 GIANINNI. Daniele. La Tutela dell'ambiente. C.f. $<$ https:// www.iusexplorer.it/Publica/FascicoloDossier/La_tutela_dell_ambi ente $/$ idDocMaster $=4065982 \&$ idDataBanks $=19 \&$ canale $=13>$. Accesso in: 27 apr. 2016.
Pure sotto quest'aspetto ha avuto grande rilievo l'interpretazione evolutiva offerta dalla giurisprudenza della Corte Costituzionale: secondo la Consulta, infatti, la tutela del paesaggio di cui all'art. 9 Cost. "deve essere intesa nel senso lato di tutela ecologica" (Corte Cost., 3 ottobre 1990, n. 430), dunque anche come "interesse alla conservazione dell'ambiente naturale" (Corte Cost., 11 luglio 1989, n. 391), mentre la tutela della salute di cui all'art. 32 Cost. vale anche come "tutela dell'ambiente in cui l'uomo vive".

Ed è proprio in ragione di una lettura congiunta degli artt. 9 e 32 Cost., quindi, che la Consulta afferma la concezione "unitaria" dell'ambiente, che comprende "la conservazione, la razionale gestione e il miglioramento delle condizioni naturali (aria, acque, suolo e territorio in tutte le sue componenti), l'esistenza e la preservazione dei patrimoni genetici terrestri e marini, di tutte le specie animali e vegetali che in esso vivono allo stato naturale e in definitiva la persona umana in tutte le sue estrinsecazioni”.

In Italia, il danno ambientale è un concetto giuridico introdotto nel 1986 dalla Legge 349/1986 nel suo articolo 18, dove si tutela "in sé per sé" costatandosi che merita risarcimento indipendentemente dalla lesione di qualsiasi altro diritto soggettivo, come la proprietà o la salute. La materia danno ambientale è disposta ora nella Parte VI della Legge 152/2006 Codice Ambiente, articolo 300, dove si definisce il danno ambientale come: “ danno ambientale s'intende che è qualunque deterioramento rilevante e misurabile, diretto o indiretto, di una risorsa naturale o utilità assicurata da quest'ultima".

La Costituzione della Repubblica Portoghese ${ }^{16}$ del 1976, ha consacrato l'esistenza di diritti e doveri costituzionali e, tra questi, il diritto all'ambiente come diritto fondamentale. Si costata, negli articoli $66^{\circ}$ e $52^{\circ}$ n. 3 della Carta, che la protezione ambientale è dovere fondamentale dello Stato portoghese. Così, la Costituzione prevede che "tutti hanno diritto a un ambiente di vita umano, sano ed ecologicamente equilibrato e il dovere di difenderlo". Si osserva, quindi, che l'Ambiente è trattato come un interesse diffuso, ossia, un interesse che possiede titolarità individuale. Il disposto sull'articolo $9^{\circ}$, lett. "e", impone, dunque, allo Stato il dovere di "e) Proteggere e valorizzare il patrimonio culturale del popolo portoghese, difendere la natura e l'ambiente, preservare le risorse naturali e assicurare un corretto ordinamento del territorio".

16 C.f. $<$ http://www.parlamento.pt/Legislacao/Paginas/ConstituicaoRepublicaPortuguesa.aspx>. Accesso in: 20 apr. 2016. 
La Costituzione Olandese ${ }^{17}$, nel testo dell'articolo 21, dispone che le autorità pubbliche hanno l'obbligo di tutelare l'abitazione del Paese e proteggere e migliorare l'ambiente.

Altre costituzioni europee hanno espresso anche la loro preoccupazione ambientale come quella della Slovacchia, del 1992 (art. 45), della Lettonia, del 1992 (art. 115), della Polonia, del 1997 (art. 74), della Federazione Russa, del 1993 (art. 42) e la Costituzione dell'Ucraina, del 1996 (art. 50-1).

\subsection{L'Ambiente nelle Costituzioni dell'America del Sud}

La Costituzione Argentina ${ }^{18}$ del 1994, nel suo articolo 41, ha garantito che le risorse naturali devono essere preservate, imponendo altresì l'educazione ambientale come strumento di sensibilizzazione conservazionista che dispone:

\footnotetext{
Articolo $41^{\circ}$ - Tutti gli abitanti godono del diritto a un ambiente sano, equilibrato, adatto allo sviluppo umano e a che le attività produttive soddisfino le esigenze del presente senza compromettere le necessità delle generazioni future, con il dovere di tutelarle. Il danno ambientale genererà in primis l'obbligo di ripristinare la situazione precedente, nel rispetto della legge.
}

Le autorità provvederanno alla tutela di tale diritto, all'utilizzo razionale delle risorse naturali, alla conservazione del patrimonio naturale e culturale e della diversità biologica, oltre che all'informazione e all'educazione ambientali.

Compete alla Nazione dettare norme che contengano i presupposti minimi di tutela e alle province dettare quelle di dettaglio, senza modificare le giurisdizioni locali.

È vietato l'ingresso nel territorio nazionale di residui realmente o potenzialmente pericolosi e di scorie radioattive.

Si registri la lezione di Adede y Castro (2003, p. 66) ${ }^{19}$ a proposito dell'art. 41:

17 COSTITUZIONE OLANDESE. C.f.<http://www.oefre. unibe.ch/law/icl/nl00000_.html>. Accesso in: 09 mag. 2016.

18 COSTITUZIONE ARGENTINA C.f. $<$ http://infoleg. mecon.gov.ar/infolegInternet/anexos/0-4999/804/norma.htm>. Accesso in: 14 mar. 2016.

19 ADEDE Y CASTRO, João Marcos. Resíduos perigosos no direito ambiental internacional: sua internalização nos países do Mercosul. Porto Alegre: Sergio A. Fabris Editor, 2003.
$\mathrm{Si}$ osserva la preoccupazione del legislatore costituzionale di ripartire competenze tra il governo nazionale e i governi provinciali, tanto che questi ultimi possono emanare norme di protezione ambientali, a condizione che non contrastino con la legislazione del Paese.

La Costituzione del Peru ${ }^{20}$, promulgata nel 1993, rende omaggio ai diritti della personalità e alla dignità della persona umana. ${ }^{21} \mathrm{Il}$ suo testo ha concesso un capitolo per trattare le questioni ambientali intitolato $\mathrm{Del}$ Ambiente y los recursos naturales (L'Ambiente e le risorse naturali) che inizia dall'articolo 66 e termina all'articolo 69. In questa Costituzione, le risorse rinnovabili e non rinnovabili sono legato della Nazione e lo Stato è sovrano nel relativo utilizzo. Assicura, ancora, l'informazione che, attraverso Legge Organica, se ne farà la concessione dell'utilizzo da parte di privati, permettendo un diritto reale di proprietà soggetto a norma legale, nel modo seguente:

\section{ARTÍCULO 66. RECURSOS NATURALES}

Los recursos naturales, renovables y no renovables, son patrimonio de la Nacion. El Estado es soberano en su aprovechamiento. Por ley organica se fijan las condiciones de su utilizacion y de su otorgamiento a particulares. La concesion otorga a su titular un derecho real, sujeto a dicha norma legal.

L'articolo 67, intitolato "Della politica ambientale", ci informa che spetta allo Stato promuovere l'uso sostenibile delle risorse naturali. È importante osservare, ancora, l'informazione riportata dall'articolo 68, concernente alla conservazione della diversità biologica nelle aree naturali, rendendo nuovamente responsabile lo Stato della promozione della conservazione di tale diversità biologica. Nel concludere il capitolo riferente all'ambiente e i suoi ecosistemi, concernente l'Amazzonia peruviana, risulta dall'articolo 69 che " $E l$ Estado promueve el desarrollo sostenible de la Amazon a con una legislacion adecuada".

La Costituzione del Cile ${ }^{22}$ (1980), invece, è stata chiara nel disporre che tutti hanno il diritto di vivere in un ambiente senza contaminazione, permettendo alla legge di restringere i diritti e le libertà individuali per proteggere l'Ambiente:

20 COSTITUZIONE DEL PERU. C.f. $<$ http://vlex.com/vid/ constitucion-politica.peru-42814763>. Accesso in: 13 apr. 2016.

21 COSTITUZIONE DEL PERU. C.f.<http://vlex.com/vid/ constitucion-politica-peru-42814763>. Accesso in: 06 mag. 2016.

22 COSTITUZIONE DEL CILE. C.f.<https://www.camara.cl/ camara/media/docs/constitucion_politica.pdf $>$. Accesso in: 13 apr. 2016. 
$8^{\circ} \mathrm{El}$ derecho a vivir en un medio ambiente libre de contaminación. Es deber del Estado velar para que este derecho no sea afectado y tutelar la preservación de la naturaleza. La ley podrá establecer restricciones específicas al ejercicio de determinados derechos o libertades para proteger el medio ambiente;

In Venezuela ${ }^{23}$ la Costituzione del 1999 ha concesso all'ambiente un trattamento giuridico specifico, inserendo il Capitolo IX (articoli 127 a 129), "Dei diritti Ambientali", tra i Diritti Umani. Tuttavia, la Costituzione elegge il recupero in natura come mezzo primordiale di riparazione per il danno ambientale, secondo espressa la Costituzione negli articoli 123 e 29, che stabiliscono le sanzioni e il Principio della Precauzione come guide della protezione ambientale, nel modo seguente:

\footnotetext{
"Le sanzioni che si destinano all'imposizione di misure che garantiscano di riportare l'Ambiente al suo stato naturale non saranno soggette ad alterazioni. Qualunque disposizione di modificazione del presente articolo dev'essere adottata da altre misure per garantire il rispristino dei danni all'Ambiente, della stessa specie e nello stesso posto, attraverso compensazione o pagamento alternativo di un ammontare relativo al valore del danno cagionato".
}

La Costituzione dello Stato Plurinazionale della Bolivia $^{24}$ ha risaltato enormemente la preservazione ambientale, prevedendo, nella sezione I del suo capitolo quinto, intitolato "Diritti Sociali ed Economici" che l'ambiente è soggetto di diritti, Dichiara il contenuto dell'articolo 33 della costituzione Politica dello Stato della Bolivia:

Le persone hanno diritto a un ambiente sano, protetto ed equilibrato. L'esercizio di questo diritto deve permettere che individui e comunità, delle generazioni presenti e future, e altri esseri viventi, si sviluppino normalmente e in modo permanente.

In Brasile ${ }^{25}$, il costituzionalismo ambientale è stato avviato nella Costituzione del 1967 che, nel suo articolo 172, ha menzionato, per la prima volta, l'Ecologia come valore. L'attuale Costituzione (1988) ha inaugurato, però, un capitolo particolarmente dedicato all'Ambiente. Ciò

23 COSTITUZIONE DE VENEZUELA. Tribunal Supremo de Justicia. C.f.<http://www.tsj.gov.ve/legislacion/constitucion1999. htm >. Accesso in: 15 mag. 2016.

24 COSTITUZIONE DELLO STATO PLURINAZIONALE DELLA BOLIVIA C.f.<http://www.ftierra.org/index.php/ generales/14-constitucion-politica-del-estado $>$. Accesso in: 05 apr. 2016.

25 COSTITUZIONE DE BRASILE.C.f.<http://www.planalto. gov.br/ccivil_03/constituicao/constituicao.htm>. Accesso in: 13 apr. 2016. nonostante, sul Capitolo "Ambiente" il testo costituzionale del 1988 ha dimostrato, espressamente, la preoccupazione verso L'Ambiente negli articoli $5^{\circ}$, LXXIII; 20, II; 23, VI; 24, VI, VII e VIII; 129, III, 220, \ $3^{\circ}$, II; 170, VI e, infine, nel 186, II, restando irrefutabile che l'Ambiente Ecologicamente Equilibrato è diritto di tutti e il dovere conservazionista è obbligo imposto allo Stato e ai cittadini.

L'Ecuador (1983), la Colombia (1991), il Paraguay (1992) e l'Uruguay (1996) hanno anche imposto allo Stato il dovere della preservazione ambientale.

\subsection{L'Ambiente nelle Costituzioni dell'America Centrale e del Nord}

In Messico ${ }^{26}$, dal 1971, le costituzioni menzionano la protezione ambientale. La Carta del 1971 concede al Congresso dell'Unione il diritto di rivedere le azioni del Potere Esecutivo per prevenire e combattere la contaminazione ambientale. La Costituzione del 1982 introduce, nell'articolo 25, il Principio dello Sviluppo Sostenibile. Nel 1987, la Costituzione dichiara, nell'articolo 27 , il diritto dello Stato d'imporre restrizioni alla proprietà privata ai fini della preservazione e rispristino ecologico e, nell'articolo 73, descrive il sistema di concorrenza delle competenze in materia ambientale tra $i$ tre livelli del governo. La Costituzione attuale, datata del 1999, nel suo paragrafo $5^{\circ}$ dell'articolo $4^{\circ}$, ha stabilito che ogni persona ha il diritto a un Ambiente adeguato per il proprio sviluppo e benessere, ai fini di proteggere gli individui come entità biologiche e sociali, così come per stabilire la protezione dell'Ambiente, considerandolo come un Diritto Sociale o di terza generazione. È interessante registrare che, partendo dalla premessa della necessità di una maggiore tutela dei beni ambientali, organizzazioni non governative ambientaliste si sono unite e hanno creato l'Accordo di Cooperazione Ambientale dell'America del Nord (Acaan), firmato parallelamente al NAFTA dagli stessi governi, ai fini di monitorare e imporre l'adempimento delle norme ambientali nazionali. In tal modo il Canada, gli Stati Uniti e il Messico prendono l'impegno di non utilizzare misure ambientali come barriere non tariffarie e di far compiere, nei loro territori, le loro rispettive legislazioni ambientali domestiche.

26 CONSTITUZIONE DEL MEXICO. C.f.<http://www.constitucion.gob.mx/>. Accesso in: 21 apr. 2016. 
In Canada ${ }^{27}$, nonostante ci sia un'enorme preoccupazione rispetto alla preservazione ambientale, la Carta Magna canadese, compresa la Dichiarazione dei Diritti e Libertà, è completamente silente in materia di protezione ambientale. Essendo così, i Tribunali stanno cercando di applicare nuove norme e principi riguardanti la legislazione ambientale. Inoltre, le azioni civili ambientali sono sempre più comuni nei Tribunali canadesi; richieste che coinvolgono denunce di perdite di sostanze chimiche, terreni contaminati, emissioni sonore e gas inquinanti sull'atmosfera provocati da grandi progetti industriali (LAWYERS, 2012) ${ }^{28}$. Anche se il Diritto Ambientale non è codificato, né esiste la previsione costituzionale di protezione all'Ambiente, c'è in Canada un Dipartimento dell'Ambiente, creato dall'atto del Ministero dell'Ambiente nel 1971. La regolazione ambientale è fatta dal citato Ministero, dall'Agenzia di Valutazione Ambientale Canadese e dalle agenzie delle province.

Negli Stati Uniti d'America, la Costituzione non è anche chiara nel menzionare la protezione ambientale, cosa comprensibile data la sua longevità. Tuttavia, nel 1969, è stata promulgata la Legge Nazionale di Politica Ambientale (National Environmental Policy Act - NEPA), che costituisce un marchio storico fondamentale, non solo perché ha proporzionato un cambiamento profondo nella politica ambientale nazionale e nella presa di decisioni del governo federale, in permanente cooperazione con i governi statali, locali e altri organizzazioni pubbliche e private, compatibilmente con le condizioni di esistenza di un'armonia produttiva tra l'uomo e la natura, il benessere generale e delle presenti e future generazioni di nordamericani, ma anche perché costituisce pioniere e notabile modello di legge ambientale, tanto dinanzi al Diritto Comparato quanto dinanzi al Diritto Internazionale. Con l'obiettivo di implementare sul territorio americano le diverse norme protettive dell'Ambiente che venivano pubblicate, l'allora presidente Richard Nixon ha instituito, il 02 dicembre del 1970, l'EPA -Environmental Protection Agency, organo federale che possiede, tra diverse attribuzioni, lo stabilimento di parametri d'inquinamento per le specifiche componenti dell'Ambiente quali aria, terra, acqua e

27 CANADA. Consolidation of the Constitution Acts, 1967 to 1982 C.f.<http://www.egov.ufsc.br/portal/sites/default/files/ anexos/21363-21364-1-PB.htm>. Accesso in: 09 mag. 2016.

28 LAWYERS, Blakes. Environment law in Canada. 2012. Disponibile in: <http://www.blakesfiles.com/Guides/2012_Blakes_Environmental_Law_in_Canada_EN.pdf>. Accesso in: 20 apr. 2016. la cui missione consiste nel proteggere la salute umana e l'ecosistema. Successivamente e armonicamente, sono state pubblicate nuove norme di protezione dell'Ambiente, tra le quali si evidenziano il Clean Air Act, il Federal Water Pollution Control Act e, l'11 dicembre del 1980, il Comprehensive Environmental Response, Compensation, and Liability Act (CERCLA), più conosciuto come Superfund, emendato il 17 ottobre del 1986 dal Superfund Amendments and Reauthorization Act $(S A R A)$, che disciplina fondamentalmente la responsabilità civile per danno all'Ambiente negli Stati Uniti.

La Costituzione della Repubblica di $\mathrm{Cuba}^{29}$ impone allo Stato il dovere di tutelare l'Ambiente primeggiando lo sviluppo economico e sociale sostenibile per innalzare la vita umana a valore fondamentale. In questo senso il suo articolo 27:

\begin{abstract}
“Artículo 27.-El Estado protege el medio ambiente y los recursos naturales del país. Reconoce su estrecha vinculación con el desarrollo económico y social sostenible para hacer mas racional la vida humana y asegurar la supervivencia, el bienestar y la seguridad de las generaciones actuales y futuras. Corresponde a los órganos competentes aplicar esta política. Es deber de los ciudadanos contribuir a la protección del agua, la atmósfera, la conservación del suelo, la flora, la fauna y todo el rico potencial de la naturaleza."
\end{abstract}

Altre Costituzioni dell'America Centrale, come quelle del Guatemala (1985) e Nicaragua (1987), hanno anche espresso la loro preoccupazione ambientale.

Si può, dunque, affermare senz'altro che le costituzioni americane, anche se dei Paesi meno fortunati, prevedono la protezione ambientale come Principio espresso.

\subsection{L'Ambiente nelle Costituzioni dell'Africa}

Nonostante ci sia uno sforzo mondiale, compreso attraverso il Fondo Francese per l'Ambiente Mondiale (FFEM), parte dell'Africa necessita ancora di protezione ambientale, sia giuridica, sia fatica. Gli esperti in desertificazione calcolano che cinquecento milioni di ettari sono affetti dal degrado del suolo, il che corrisponde al 65\% dell'area prima destinata all'agricoltura nel continente.

29 COSTITUZIONE DELLA REPUBBLICA DI CUBA. C.f. $<$ http://www.cuba.cu/gobierno/cuba.htm>. Accesso in: 09 mag. 2016. 
La Costituzione di Angola ${ }^{30}$ del 2010, assicura, nel suo articolo 21. ${ }^{\circ}$, voce 1 , quando tratta dei compiti fondamentali dello Stato: “m) Promuovere lo sviluppo armonioso e sostenibile in tutto il territorio nazionale, proteggendo l'ambiente, le risorse naturali e il patrimonio storico, culturale e artistico nazionale". Tuttavia, l'articolo 39. informa che "Tutti hanno il diritto di vivere in un ambiente sano e non inquinato, come pure hanno il dovere di difendere e preservarlo". Anche la Costituzione angolana, però, impone allo Stato e ai privati l'adozione di misure necessarie alla protezione ambientale (articolo $21{ }^{\circ}$, voce 2 ); la limitazione del diritto di andare e venire quando quest'esercizio degrada l'Ambiente (articolo 46..$^{\circ}$ ); il diritto di postulare, presso il Potere Giudiziario, attraverso azione popolare, la protezione ambientale. (articolo $74 .^{\circ}$ ); la restrizione delle attività economiche che degradano l'Ambiente (articolo 89. ${ }^{\circ}$, voce 1) e la consacrazione del Principio dello Sviluppo Sostenibile (Articolo 91..$^{\circ}$ ).

Nel Mozambico ${ }^{31}$, la Costituzione del 2004 è chiara nel determinare, nel suo articolo 45, f, che "Ogni cittadino ha il dovere di "difendere e conservare l'ambiente". Inoltre, gli concede, ai sensi dell'articolo 81, b, di Azione Popolare, il diritto di portare a giudizio per garantire "il diritto di promuovere la prevenzione, la cessazione o il perseguimento giudiziale delle infrazioni contro la salute pubblica, i diritti dei consumatori, la preservazione dell'ambiente e il patrimonio culturale". Dispone ancora:

\begin{abstract}
Articolo 90 (Diritto all'ambiente)
Ogni cittadino ha il diritto di vivere in un ambiente equilibrato e il dovere di difenderlo.

Lo Stato e le autarchie locali, con la collaborazione delle associazioni di difesa dell'ambiente, adottano politiche di difesa dell'ambiente e velano per l'utilizzazione nazionale di tutte le risorse naturali.

Articolo 117 (Ambiente e qualità della vita) 1. Lo Stato promuove iniziative per garantire l'equilibrio ecologico e la conservazione e preservazione dell'ambiente mirando a migliorare la qualità della vita dei cittadini. 2. Ai fini di garantire il diritto all'ambiente nell'ambito di uno sviluppo sostenibile, lo Stato adotta politiche mirando a: a) prevenire e controllare l'inquinamento e l'erosione; b) integrare gli obiettivi ambientali nelle politiche settoriali; c)
\end{abstract}

30 COSTITUZIONE DELLA REPUBBLICA DI ANGOLA. C.f.<http://www.governo.gov.ao/Arquivos/Constituicao_da_Republica_de_Angola.pdf $>$. Accesso in: 20 apr. 2016.

31 COSTITUZIONE DELLA REPUBBLICA DI MOÇAMBIQUE. C.f. $<$ http://www.presidencia.gov.mz/files/republica/constituicao_republica_moc.pdf >. Accesso in: 21 apr. 2016. promuovere l'integrazione dei valori dell'ambiente nelle politiche e programmi educativi; d) garantire l'utilizzo razionale delle risorse naturali nella protezione della capacità di rinnovazione, della stabilità ecologica e dei diritti delle generazioni future; e) promuovere l'ordinamento del territorio ai fini di un corretto luogo di attività e di uno sviluppo socio-economico equilibrato.

In Africa del Sud ${ }^{32}$, la Costituzione datata del 1996, dispone, nel suo articolo 24, che ogni persona ha diritto a un ambiente sano per il suo benessere, e rende chiaro omaggio al Principio della Solidarietà Intergenerazionale, al Principio della Prevenzione e al Principio dello Sviluppo Sostenibile. Nell'articolo 146, la Costituzione stabilisce la prevalenza del proprio testo sulle norme provinciali, determinandone l'osservanza obbligatoria da parte dei legislativi locali. Infine, reitera 1'articolo 152, il Principio dello Sviluppo Sostenibile, nel determinare che i governi locali favoriscano lo sviluppo economico senza dimenticare la preservazione ambientale.

Si conclude, quindi, che oggi esiste l'interesse verso le questioni ambientali e che le legislazioni africane sono in costante evoluzione in cerca di perfezionamento giuridico per una migliore tutela dell'Ambiente.

\subsection{L'Ambiente nelle Costituzioni dell'Asia}

Sul documento intitolato Asia-Pacific Perspectives on Environmental Ethics dell'UNESCO, è possibile verificare la preoccupazione dei Paesi asiatici per l'etica ambientale e, di conseguenza, si fa una riflessione sui modi di vita e i suoi effetti sulla natura. In quest'opera c'è un articolo di un ingegnere giapponese, Masato Motoki, il quale afferma che i problemi ambientali sono noti ai giapponesi e che, però, esiste la difficoltà di come affrontare tali questioni ${ }^{33}$.

Nonostante la Costituzione del Giappone ${ }^{34}$ del 1946 non menzioni la tutela ambientale, è importante registrare che il governo giapponese è sensibile alle questioni ambientali, come si deduce da questo brano tratto dal

32 COSTITUZIONE DEL AFRICA DE SUL. C.f.<http:// www.jurisciencia.com/vademecum/constituicoes-estrangeiras/ constituicao-da-africa-do-sul-constitution-of-the-republic-of-southafrica/136>. Accesso in: 20 apr. 2016.

33 MOTOKI, Masato. Observations on environmental ethics, animal rights and culture. In: ASIAPACIFIC perspectives on environmental ethics. Bangkok: UNESCO Bangkok, 2008.p. 71.

34 COSTITUZIONE GIAPPONE. C.f.<http://www.br.embjapan.go.jp/cultura/pdf/constituicao.pdf $>$. Accesso in: 20 apr. 2016. 
sito dell'Ambasciata del Giappone in Brasile ${ }^{35}$ :

L'inquinamento ambientale in Giappone accompagna l'industrializzazione dall'Era Meiji (1868-1912). Negli anni '60, malattie causate dall'acqua contaminata delle fabbriche e dall'inquinamento dell'aria sono sorte in diverse aree del Giappone. Le rigide misure di protezione ambientale implementate subito dopo sono riuscite a ridurre l'inquinamento causato da queste emissioni. Tuttavia, problemi importanti devono ancora essere risolti con azioni necessarie per, ad esempio, ridurre le emissioni dei gas di effetto serra e di materiale particolato e ampliare il riciclaggio dei residui industriali e domestici. Questioni ambientali globali, come la distruzione dello strato di ozono e il riscaldamento globale non possono essere risolti dai singoli Paesi, ed è evidente che la cooperazione di tutti i Paesi è sempre più necessaria per proteggere l'ambiente. IL Giappone esercita un ruolo attivo in questo sforzo globale.

La Terza Sessione della Conferenza delle Parti della Convenzione Quadro delle Nazioni Unite sui Cambiamenti Climatici (COP3) è stata realizzata a Kyoto nel dicembre del 1997 e ha adottato il Protocollo di Kyoto per regolare le emissioni di diossine e altri gas di effetto serra nel periodo compreso tra il 2008 e il 2012. Il Giappone ha ratificato il protocollo nel giugno del 2002. Nonostante gli Stati Uniti abbiano rifiutato di ratificarlo, la ratificazione della Russia, nel 2004, ha significato che i criteri necessari per l'implementazione formale del protocollo erano stati soddisfatti e il trattato è entrato in vigore nel febbraio del 2005. Il Giappone promuove diverse iniziative, in uno sforzo per creare una "società a basso carbonio" di economia e Ambiente sani.

Inoltre, di fronte alle enormi catastrofi ambientali subite dal Paese, diverse misure legislative sono state prese secondo l'Ambasciata del Giappone ${ }^{36}$ :

La regolazione sulle emissioni di fuliggine e fumo, che è entrata in vigore nel 1962, è stata incorporata alla Legge di Controllo dell'Inquinamento dell'Aria nel 1968. La Legge di Conservazione della Qualità dell'Acqua e la Legge di Controllo dell'Acqua Scaricata dalle Fabbriche, ambedue del 1958, sono state integrate alla Legge di Controllo dell'Inquinamento dell'Acqua nel 1970. La Legge Fondamentale di Misure Contro l'Inquinamento, approvata nel 1967, ha cercato di creare principi e politiche comuni per il controllo dell'inquinamento in tutte le agenzie del governo e promuovere uno sforzo integrato per pulire l'Ambiente. La

35 AMBASCIATA DEL GIAPPONE IN BRASILE. C.f.<http://www.br.emb-japan.go.jp/cultura/ambiente.html>. Accesso in: 14 mag. 2016.

36 AMBASCIATA DEL GIAPPONE IN BRASILE. C.f.<http://www.br.emb-japan.go.jp/cultura/ambiente.html>. Accesso in: 14 mag. 2016.
Legge Fondamentale indica le responsabilità del governo centrale, governi locali e imprese rispetto al controllo dell'inquinamento. Inoltre, la Legge Fondamentale ha creato il quadro di lavoro per stabilire parametri di qualità ambientale, creare programmi di controllo dell'inquinamento e ausiliare le vittime delle malattie causate dall'inquinamento. Nel 1972, il concetto di responsabilità consensuale per indennizzi, che conferisce responsabilità alle aziende per i problemi di salute, accidentali o no, derivanti dall'inquinamento è stato introdotto in diverse leggi correlate.

Nel 1993, la Legge Basica per il Controllo dell'Inquinamento Ambientale è stata sostituita dalla Legge Ambientale Basica, che è stata approvata per facilitare l'implementazione di misure ampie e sistematiche per proteggere l'Ambiente. Secondo questa nuova Legge Basica, il Giappone sta lavorando attivamente per promuovere la preservazione ambientale intorno al mondo attraverso la cooperazione internazionale e una rivalutazione dell'alto livello di consumo praticato dalla società. Nel 1997, la Legge di Valutazione dell'Impatto Ambientale è entrata in vigore. Questa legge definisce parametri per valutare l'impatto ambientale dei progetti di larga scala del settore pubblico e privato.

Nel 2001, l'Agenzia Ambientale, che era stata creata nel 1971, è stata promossa a livello ministeriale, diventando il Ministero dell'Ambiente.

La Legge sulle Misure Speciali contro la Diossina è entrata in vigore nel 1999. Questa legge includeva provvisioni per regolare le emissioni di diossina, monitorare gli effetti della sostanza nella salute e nell'Ambiente e preparare i piani governativi per ridurre le emissioni.

Insoddisfatti col ritmo degli sforzi del governo nazionale per ridurre l'inquinamento dell'aria, nel 2003 le provincie di Tokio, Saitama, Chiba e Kanagawa hanno implementato regole ancora più rigide coprendo le emissioni di materiali particolati di camion a diesel e autobus. Le vetture che non rispettano le nuove norme devono essere sostituiti o devono istallare filtri speciali.

In $\mathrm{Cina}^{37}$, l'Ambiente è previsto dalla Costituzione della Repubblica Popolare, del 1982, nei suoi articoli 9 e 26, che annunciano rispettivamente:

Articolo 9 - Risorse minerali, acque, foreste, montagne, praterie, riserve, spiagge e altre risorse naturali appartengono allo Stato, ossia, a tutto il popolo, tranne le foreste, montagne, praterie, riserve e spiagge appartenenti a entità collettive secondo la legge. Lo Stato garantisce l'utilizzo razionale delle risorse, specie rare di piante e di animali. L'appropriazione o danno delle risorse naturali da

37 CINA COSTITUZIONE. C.f.<http://bo.io.gov.mo/ bo/i/1999/constituicao/index.asp>. Accesso in: 13 apr. 2016. 
parte di qualunque organizzazione o individuo per qualsiasi mezzo sono proibiti.

Articolo 26- Lo Stato protegge e implementa l'ambiente naturale ed ecologico, e previene e controlla l'inquinamento e altre minacce pubbliche. Lo Stato organizza e incoraggia la riforestazione e la protezione delle foreste.

I Tribunali dell'India rendono omaggio all'Ambiente equilibrato e sano, visto che si costata dalla Costituzione della Repubblica dell'India ${ }^{38}$, negli articoli 48A e 49, la consacrazione dell'Ambiente come bene fondamentale (BANERJEE, 2008) ${ }^{39}$ :

Art. $48^{\mathrm{a}}$ - Protezione e miglioramento dell'Ambiente e protezione delle foreste e della vita selvatica.

Art. 49- Protezione dei monumenti, luoghi e oggetti d'importanza nazionale.

Si registri che in India, nonostante il degrado del suo Ambiente ci sia noto, soprattutto quello urbano, esiste la Legge di Protezione Ambientale, promulgata nel 1986, che è considerata una direttrice per lo sfruttamento di tutte le risorse naturali. Inoltre, sono state approvate diverse leggi ambientali, come la Legge di Combattimento all'Inquinamento Atmosferico (1987), la Legge di Protezione delle Acque (1988) e la Legge di Gestione dei Rifiuti Solidi Pericolosi (1989), tra altre.

Singapore è, senz'altro, uno dei migliori esempi di Sviluppo Sostenibile efficiente, sebbene non ci sia un precetto costituzionale protettivo dell'Ambiente nella sua Carta del 1963. La città-nazione è stata capace di trasformare una zona completamente deteriorata in un posto dove si costata una delle migliori qualità ambientali del mondo, come sostiene Tan Tian Chong, direttore di Sviluppo Tecnologico della Building and Construction Authority (BCA) ${ }^{40}$ : "Abbiamo svolto un esteso insieme di azioni che passa, necessariamente, attraverso la creazione del Green Building Masterplan (guida di parametri di sostenibilità) e la concessione del BCA Green Mark (certificato concesso ai progetti approvati)", spiega. Secon-

38 INDIA LA COSTITUZIONE.. C.f.<http://lawmin.nic.in/ olwing/coi/coi-english/coi-indexenglish.htm>. Accesso in: 20 apr. 2016.

39 BANERJEE, Debadyuti. Environmental Jurisprudence in India: a look at the initiatives of the supreme court of india and their success at meeting the needs of eviro-social justice. 2008. Disponibile in: $<$ http://www.academia.edu/430162/EnvironmentaljurisprudenceinIndialookattheinitiativesoftheSupremeCourtofIndiaandtheir_ success_at_meeting_the_needs_of_enviro-social_justice $>$. Accesso in 20 apr. 2016.

40 SINGAPORE COSTITUZIONE. C.f.<https://www.bca. gov.sg/>. Accesso in: 20 apr. 2016. do il dirigente, il numero di edifici "verdi" a Singapore, dal 2005, è aumentato da 17 a più di 940 unità. "Questo si traduce in oltre 28 milioni di metri quadri di area costruita conformemente agli standard di efficienza energetica, riutilizzo dell'acqua e protezione ambientale. Il certificato è stato richiesto da 140 edifici in Cina, Malesia, India e Arabia Saudita, tra altri Paesi”, aggiunge.

A Singapore, ci sono anche importanti progetti di efficienza energetica e di trattamento dell'acqua. Per i primi, l'Agenzia Nazionale dell'Ambiente (National Environment Agency - NEA) ha lanciato, nel novembre del 2008, il programma Greet - Grant for Energy Efficient Technologies (Fondo per la Concessione di Tecnologie di Efficienza Energetica), che mira a incentivare le industrie locali a investire nelle tecnologie per ridurre il consumo di energia. Per convincere le imprese ad aderire al programma - nella loro maggioranza entità dei settori farmaceutico, chimico, elettronico e di costruzione navale -, si è impiegata la stessa strategia usata dalla BCA: credito facile. Oggi, l'iniziativa finanzia fino al 50\% dei costi di adeguazione (limitati a US\$ 2 milioni per progetto), che includono l'acquisto di equipaggiamenti, la qualificazione della mano d'opera e l'assistenza tecnica ${ }^{41}$.

In definitiva, quindi, il costituzionalismo contemporaneo elegge l'Ambiente come Diritto Fondamentale, consuetamente, disponendo, nelle costituzioni scritte, il dovere dello Stato e di tutti i cittadini della sua preservazione.

\subsection{L'Ambiente nelle Costituzioni dell'Oceania}

La Costituzione dell'Australia ${ }^{42}$, datata del 1900, non menziona nulla sulla protezione ambientale, il che non sorprende, data l'epoca della sua promulgazione quando, com'è già stato detto, non constava ancora nelle Carte Magne tale preoccupazione, soprattutto a causa della poca degradazione ambientale coniugata alla scarsa conoscenza degli effetti dell'attuazione umana nel deterioramento dell'Ambiente. Comunque, in un documento ufficiale del Governo, datato del 2010, si riconosce che, nonostante la Costituzione australiana non accenni all'Ambiente, esso è di somma importanza ai precetti giuridici della nazione:

41 PETROBRÁS. C.f.<http://www.petrobras.com/pt/magazine/post/cingapura.htm>. Accesso in: 4 mag. 2016.

42 AUSTRALIA COSTITUZIONE. <http://www.egov.ufsc. $\mathrm{br} / \mathrm{portal} / \mathrm{sites} /$ default/ files/anexos/21382-21383-1-PB.htm>. Accesso in: 20 apr. 2016. 
This list of powers given to the Commonwealth Parliament does not expressly refer to a number of important subjects including education, the environment, criminal law, and roads - but this does not mean that those subjects are wholly outside the Parliament's powers. For example, even though the Commonwealth Parliament has no specific power in relation to the environment, it can, under its external affairs power, prohibit the construction of a dam by a State if that is necessary to give effect to an international agreement on the environment. The legislative powers of the Commonwealth Parliament can also be expanded by the Parliaments of the States referring matters to the Commonwealth Parliament under section 51(XXXVII).

In Nuova Zelanda la Costituzione è formata da vari documenti e, soprattutto, dalla Carta del 1986, non esistendo, quindi, un documento formale. Per quanto riguarda la gestione delle questioni ambientali, tutte le autorità, sia a livello federale, sia a livello locale sono obbligate a seguire le direttrici stabilite dal documento intitolato Valutazione Ambientale Strategica, in cui si verifica l'enorme valorizzazione ambientale conferita dal Paese e dalla sua comunità scientifica.

Così, sebbene sia il più giovane dei continenti, lì l'ambiente è estremamente protetto, anche se non espressamente sui testi, a causa della tradizione della Common Law, ma, senz'altro, la Scienza Giuridica se ne preoccupa e stabilisce delle norme protettive.

\section{RESPONSABILITÀ PER DANNI ALL'AMBIENTE NEL DIRIITO COMUNITARIO}

Presentando il panorama mondiale, soprattutto europeo, dove è chiaro che il Diritto Costituzionale contemporaneo ha eletto l'Ambiente come Diritto Umano, si rende doverosa l'imputazione di responsabilità a chi lo degrada.

In questo senso, il Diritto Europeo, attraverso la Direttiva 2004/35, impone ai Paesi membri l'osservanza di norme generali che guidano la costruzione di regole giuridiche interne per la corretta imputazione di responsabilità a quelli che deteriorano l'ambiente, imperando la prevenzione e la riparazione dei danni ambientali.

Occorre sottolineare che, dopo tutto, le direttive e i regolamenti sono elementi del corpo normativo del Diritto dell'Unione Europea:

Le direttive europee fissano agli Stati membri gli obiettivi da raggiungere, delegandogli la scelta dei mezzi per farlo, potendo avere come destinatari uno o vari Stati membri, oppure la loro totalità. Perché i principi stabiliti nelle direttive producano effetti a livello del cittadino, il legislatore nazionale deve adottare un atto di trasposizione, al diritto nazione, degli obiettivi definiti nella direttiva. ${ }^{43}$

Rispetto alla loro applicabilità e incorporazione nell'ordinamento giuridico nazionale degli Stati membri,

\begin{abstract}
Le direttive prevedono una data limite perché siano trasposte al diritto nazionale: gli Stati membri dispongono, per la trasposizione, di un margine di manovra che gli permette di considerare le specificità nazionali. La trasposizione deve essere compiuta entro il termine stabilito dalla direttiva.
\end{abstract}

È importante evidenziare che la direttiva rafforza il progetto comune d'integrazione e di armonizzazione della legislazione e, secondo il Diritto Comunitario, si può affermare che "le direttive sono utilizzate per armonizzare le legislazioni nazionali, in particolare al fine della realizzazione del mercato unico (ad esempio le norme riguardanti la sicurezza dei prodotti)."

Partendo dall'individuazione riguardante l'esistenza di "molti siti contaminati che suscitano rischi significativi alla salute e la perdita della biodiversità", così come dell'inazione che risulta nell'aumento della contaminazione e nella futura perdita della biodiversità" e, considerando l'effettività della prevenzione e della riparazione dei danni ambientali "mediante l'applicazione del principio dell'inquinatore-pagatore, previsto dal Trattato", la direttiva in questione mira a responsabilizzare finanziariamente l'operatore la cui attività abbia causato danni ambientali o la minaccia imminente di tali danni, al fine di indurre gli operatori ad adottare misure e a svolgere pratiche in modo da ridurre i rischi dei danni ambientali”.

Secondo l'intendimento della stessa Direttiva (2004/35), "I danni ambientali includono ugualmente quelli causati dall'inquinamento atmosferico, nella misura in cui essa danneggia l'acqua, il suolo, le specie o gli habitat naturali protetti".

In questo modo, si raccomanda che la decisione concernente alla riparazione dei danni ambientali consideri le condizioni locali, senza dimenticare che sia la riparazione, sia la prevenzione, devono essere in consonanza con il "Principio dello Sviluppo Sostenibile", come gli rende giustizia il o comma $2^{\circ}$ della citata Direttiva.

43 EUROPEAN COMISSION. C.f.<http://ec.europa.eu/eu_ law/directives/directives_pt.htm>. Accesso in: 28 mag. 2016. 
Come si osserva chiaramente, la Direttiva in questione, per evitare qualunque ambiguità riguardante la buona interpretazione all'applicazione della nozione di danni ambientali proveniente da altra legislazione comunitaria rilevante, risalta l'importanza di utilizzare "criteri comuni e promuovere un'applicazione uniforme."

Attento a ciò, sorge nella Direttiva 2004/35, nel suo articolo $7^{\circ}$, 1 , la determinazione che gli operatori indentifichino "potenziali misure di riparazione, presentandole poi all'autorità competente per approvazione [...]." Spettagli, in questo caso, "decidere quali misure di riparazione applicare, nei sensi dell'Allegato II e, se necessario, con la cooperazione dell'operatore in causa"

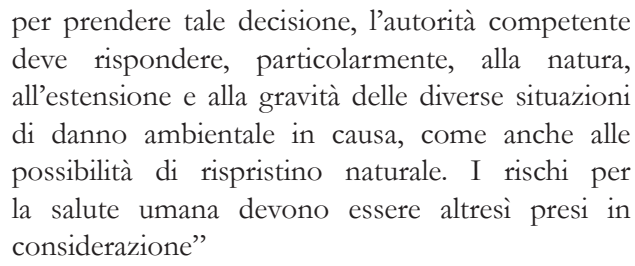

Occorre sottolineare anche che

Rispetto ai danni ambientali, la presente direttiva deve applicarsi alle attività lavorative che presentino rischi alla salute umana o all'ambiente. Tali attività devono, in principio, essere identificate per essere riferite alla legislazione comunitaria pertinente, che prevede requisiti regolamentari rispetto a certe attività $o$ pratiche considerate un rischio potenziale o reale alla salute umana o all'ambiente."

È opportuno evidenziare, conforme quello che è già stato descritto circa la prevenzione e riparazione dei danni ambientali, che la Spagna, essendo uno Stato membro dell'Unione Europea, è costretta al controllo dell'applicazione del Diritto dell'Unione Europea, compreso il Diritto Europeo dell'ambiente, in vista dell'utilizzazione di criteri comuni e della promozione di un'applicazione uniforme della Direttiva.

Inoltre, l'art. $16^{\circ}$ della sopramenzionata Direttiva, sottolinea il rapporto con il diritto nazionale, una volta che la stessa

\footnotetext{
Il ripristino dell'ambiente dovrebbe avere luogo in maniera efficace garantendo il conseguimento degli obiettivi di riparazione. A tal fine si dovrebbe definire un quadro comune la cui corretta applicazione dovrebbe essere controllata dall'autorità competente.
}

Altresì, rispetto all'esecuzione, si osserva che la Direttiva 2004/35 permette agli Stati membri di "mettere in vigore le disposizioni legislative, regolamentari e amministrative necessarie al compimento della presente"
[...], così come di "comunicare alla Commissione il testo delle principali disposizioni di diritto interno che saranno approvate nelle materie regolate" (dalla Direttiva).

Tuttavia, si risalta che l'applicazione della Direttiva è vincolata alle attività lavorative che possano causare danni o minaccia imminente di danni, nel caso ci sia sufficiente probabilità di danno ambientale in un futuro prossimo. In questo caso, la responsabilità è oggettiva, per i danni o per la minaccia di danni che l'attività dell'operatore possa causare all'acqua, al suolo, alle specie e habitat naturali protetti, come stipolato sull'allegato II, concernente alla riparazione dei danni ambientali. L'obbiettivo della riparazione primaria è rispristinare le risorse naturali e/o i servizi danneggiati o accostarle alla condizione originale.

È da segnalare che, oltre alla riparazione primaria, ci sono anche le riparazioni supplementari e di compensazione, in una zona alternativa, mentre si attende la riparazione.

Si deve registrare, però, che l'autorità che determina l'importanza dei danni considerati significativi è di competenza di ciascuno Stato membro dell'Unione Europea.

Ancora su questo punto, apoggiandoci su Souza e Yamaguchi (2011, p. 50), vale evidenziare che i seguenti principi fondamentano la politca ambientale dell'Unione Europea ${ }^{44}$ :

\section{- Il principio di precauzione: devono essere anticipati ed evitati danni all'ambiente dall'inizio. \\ - Il principio di origine: danni devono essere combattuti soprattutto alla loro origine. \\ - Il principio di chi causa: chi causa danni all'ambiente è responsabile per l'impedimento dall'inizio o per l'eliminazione dei danni causati. \\ Ci sono altri principi, più attuali, che sono i seguenti: - Il principio di sussidiarietà: il livello di azione dipende dal tipo di danno, estensione, ecc. \\ - Il principio d'integrazione: aspetti ambientali devono essere integrati in tutte le altrettante politiche dell'Unione Europea.}

Secondo Bedran e Mayer (2013, p.77), il 21 aprile del 2004, il Parlamento Europeo, attraverso la Direttiva

44 SOUZA, Mauro César Martins de; YAMAGUCHI, Taylla Evellyn. França: a construção do direito ambiental em um país desenvolvido. TÓPOS, v. 5, n. 2, p. 47-66, 2011. Accesso in: <http://revista.fct.unesp.br/index.php/topos/article/viewFile/2284/2089>. Accesso in: 24 apr. 2016. 
2004/35, ha stabilito delle direttrici volte agli Stati membri dell'Unione Europea sulla responsabilità ambientale in termini di prevenzione e riparazione dei danni ambientali.

I sistemi di responsabilità ambientale dei diversi Stati membri dell'Unione Europea variavano molto rispetto alla responsabilizzazione per i danni ambientali e alle esigenze di riparazione. Nel 2004, l’Unione Europea ha cercato di istituire un regime più uniforme di prevenzione e riparazione dei danni ambientali attraverso l'adozione della rilevante Direttiva 2004/35, circa la responsabilità ambientale in termini di prevenzione e riparazione dei danni ambientali. L'innovatrice legislazione stabilisce, per la prima volta nell'Unione Europea, un regime di responsabilità globale per i danni ambientali fondamentato sul principio dell'inquinatore- pagatore.

La protezione delle risorse naturali europee (specie e habitat protetti, acqua e suolo) e dei servizi dell'ecosistema che ne sono proporzionati è, di solito, riconosciuta come fattore importante perché l'economia di uno Stato e perché la società si sviluppi in maniera salutare.

La Direttiva stabilisce una distinzione tra due tipi di operatori: quelli coinvolti nelle attività lavorative pericolose, elencate nel suo allegato III $^{45}$, e quelli legati a

45 ALLEGATO III AT'TIVITÀ DI CUI ALL'ARTICOLO 3, PARAGRAFO 1 1. Funzionamento d'impianti soggetti ad autorizzazione, conformemente alla direttiva 96/61/CE del Consiglio, del 24 settembre 1996, sulla prevenzione e la riduzione integrate dell'inquinamento. Include tutte le attività elencate nell'allegato I della direttiva 96/61/CE, ad esclusione degli impianti o parti di impianti utilizzati per la ricerca, lo sviluppo e la sperimentazione di nuovi prodotti e processi. 2. Operazioni di gestione dei rifiuti, compresi la raccolta, il trasporto, il recupero e lo smaltimento di rifiuti e di rifiuti pericolosi, nonché la supervisione di tali operazioni e i controlli successivi sui siti di smaltimento, soggetti ad autorizzazione o registrazione, conformemente alle direttive del Consiglio 75/442/CEE, del 15 luglio 1975, relativa ai rifiuti e 91/689/CEE, del 12 dicembre 1991, relativa ai rifiuti pericolosi . Tali operazioni comprendono tra l'altro la gestione di siti di discarica ai sensi della direttiva del Consiglio 1999/31/CE, del 26 aprile 1999, concernente le operazioni di discarica di rifiuti, e il funzionamento di impianti d'incenerimento ai sensi della direttiva 2000/76/CE del Parlamento europeo e del Consiglio, del 4 dicembre 2000, sull'incenerimento di rifiuti. Ai fini della presente direttiva gli Stati membri possono decidere che tali operazioni non comprendono lo spargimento, per fini agricoli, di fanghi di depurazione provenienti da impianti di trattamento delle acque reflue urbane, trattati secondo una norma approvata. 3. Tutti gli scarichi nelle acque interne superficiali che siano soggetti ad autorizzazione preventiva conformemente alla direttiva 76/464/CEE del Consiglio, del 4 maggio 1976, concernente l'inquinamento provocato da certe sostanze pericolose scaricate nell'ambiente idrico della Comunità. 4. Tutti gli scarichi di sostanze nelle acque sotterra- tutte le altre attività occupazionali. Ciascun tipo dà origine a un livello di responsabilità diversa. Agli operatori coinvolti nelle attività elencate nell'allegato III, si applica un regime di responsabilità obiettiva. Questo significa che non è necessario determinare la colpa perché l'operatore sia responsabilizzato per i danni causati al

nee che siano soggetti ad autorizzazione preventiva conformemente alla direttiva 80/68/CEE del Consiglio, del 17 dicembre 1979, concernente la protezione delle acque sotterranee dall'inquinamento provocato da certe sostanze pericolose. 5. Lo scarico o l'immissione di inquinanti nelle acque superficiali o sotterranee che sono soggetti a permesso, autorizzazione o registrazione conformemente alla direttiva 2000/60/CE.6. Estrazione e arginazione delle acque soggette ad autorizzazione preventiva conformemente alla direttiva 2000/60/ CE.7. Fabbricazione, uso, stoccaggio, trattamento, interramento, rilascio nell'ambiente e trasporto sul sito di: a) sostanze pericolose definite nell'articolo 2, paragrafo 2 della direttiva 67/548/CEE del Consiglio, del 27 giugno 1967, concernente il ravvicinamento delle disposizioni legislative, regolamentari ed amministrative relative alla classificazione, all'imballaggio e all'etichettatura delle sostanze pericolose; b) preparati pericolosi definiti nell'articolo 2, paragrafo 2 della direttiva 1999/45/CE del Parlamento europeo e del Consiglio, del 31 maggio 1999, concernente il ravvicinamento delle disposizioni legislative, regolamentari ed amministrative degli Stati membri relative alla classificazione, all'imballaggio e all'etichettatura dei preparati pericolosi ; c) prodotti fitosanitari definiti nell'articolo 2 , paragrafo 1 della direttiva 91/414/CEE del Consiglio, del 15 luglio 1991, relativa all'immissione in commercio dei prodotti fitosanitari; d) biocidi definiti nell'articolo 2, paragrafo 1, lettera a) della direttiva 98/8/CE del Parlamento europeo e del Consiglio, del 16 febbraio 1998, relativa all'immissione sul mercato dei biocidi in quantitativi superiori.8. Trasporto per strada, ferrovia, navigazione interna, mare $\mathrm{o}$ aria di merci pericolose o di merci inquinanti definite nell'allegato A della direttiva 94/55/CE del Consiglio, del 21 novembre 1994, concernente il ravvicinamento delle legislazioni degli Stati membri relative al trasporto di merci pericolose su strada, o nell'allegato della direttiva 96/49/CE del Consiglio, del 23 luglio 1996, per il ravvicinamento delle legislazioni degli Stati membri relative al trasporto di merci pericolose per ferrovia, o definite nella direttiva 93/75/CEE del Consiglio, del 13 settembre 1993, relativa alle condizioni minime necessarie per le navi dirette a porti marittimi della Comunità o che ne escono e che trasportano merci pericolose o inquinanti. 9. Funzionamento d'impianti soggetti ad autorizzazione, conformemente alla direttiva 84/360/CEE del Consiglio, del 28 giugno 1984, concernente la lotta contro l'inquinamento atmosferico provocato dagli impianti industriali (4) relativamente al rilascio nell'aria di una qualsiasi delle sostanze inquinanti coperte da detta direttiva. 10. Qualsiasi uso confinato, compreso il trasporto di microrganismi geneticamente modificati definiti nella direttiva 90/219/CEE del Consiglio, del 23 aprile 1990, sull'impiego confinato di microrganismi geneticamente modificati. 11. Qualsiasi rilascio deliberato nell'ambiente, trasporto e immissione in commercio di organismi geneticamente modificati definiti nella direttiva 2001/18/CE del Parlamento europeo e del Consiglio (6). 12. Qualsiasi spedizione transfrontaliera di rifiuti all'interno dell'Unione europea, nonché in entrata e in uscita dal suo territorio, che necessiti di un'autorizzazione o sia vietata ai sensi del regolamento (CEE) n. 259/93 del Consiglio, del 1 febbraio 1993, relativo alla sorveglianza e al controllo delle spedizioni di rifiuti all'interno della Comunità europea, nonché in entrata e in uscita dal suo território. 
suolo, alle acque, agli habitat e alle specie protette.

A tutti gli altri operatori non legati alle attività elencate nell'allegato III, è applicabile un regime di responsabilità basata sulla colpa. In questo caso è necessario determinare la colpa o la negligenza perché all'operatore possa essere data la responsabilità.

Sono incluse nelle attività previste dall'allegato III, le attività lavorative (a fini o no di lucro) che esigano una licenza nel dominio della prevenzione e del controllo integrati dell'inquinamento; che esigano un'autorizzazione/ licenza di trattamento di residui; che effettui scariche nel mezzo acquatico, utilizzi o trasposti sostanze pericolose quali, ad esempio, prodotti chimici; che sia collegata alle captazioni d'acqua; che utilizzi, trasformi, rilasci sostanze o preparati pericolosi, prodotti fitofarmaceutici o prodotti biocidi; dispersi intenzionalmente organismi energeticamente modificati nell'ambiente, trasporti residui, produca scarti d'estrazione mineraria, coinvolga la cattura e lo stoccaggio del carbonio.

Tali attività hanno una responsabilità oggettiva per i danni o per la minaccia imminente dei danni che possano causare all'acqua, al suolo e alle specie protette di animali e piante, così come ai loro habitat naturali ${ }^{46}$.

Questo significa che non è necessario determinare l'esistenza di colpa o di negligenza da parte sua perché sia responsabile dell'adozione di misure di prevenzione e riparazione, come anche del pagamento dei rispettivi costi.

Rilevante il riconoscimento vincolativo delle direttive provenienti dal Parlamento Europeo in attività legiferante con il Consiglio dell'Unione Europea, le quali stabiliscono scopi precisi da essere compiuti dai Paesi appartenenti all'Alleanza. Tali direttrici attuano come mandati di aggiustamento legale delle nazioni europee e rendono esplicito termini a quo di compliance, cioè, impongono natura cogente ai programmi dell'Unione Europea in diversi campi. In questo senso, si utilizza, ancora una volta, una correlazione con gli insegnamenti di Marcelo Neves (2009), giacché la legislazione di carattere programmatico trabocca, secondo l'autore, una realtà erronea nella maggioranza dei Paesi. Tuttavia, nell'ambito europeo, l'esame di qualche diniego nelle legislazioni domestiche si potrebbe concepire come attività legislativa innocua, svuotata di pretesto regolatore

46 Direttiva di Responsabilità Ambientale 2004/35. societario $^{47}$.

Certamente la preservazione ambientale, spesso, si dissocia dal lucro facile, nella misura in cui s'impongono investimenti che attenuino il degrado, esigendo, quindi, dall'imprenditore la riduzione dei suoi margini di lucro e l'omaggio al Principio della Solidarietà Intergenerazionale, come sostiene Gianluca Senatore:

Creare una società fondata sull'inclusione sociale tenendo conto della solidarietà tra le generazioni e all'interno delle stesse, nonché garantire e migliorare la qualità della vita dei cittadini quale presupposto per il benessere duraturo delle persone;

In questo senso, la Costituzione Europea del 2004 quando tratta il tema con un focus sui Diritti Umani:

Unita nella diversità', l'Europa offre ai suoi popoli le migliori possibilità di proseguire, nel rispetto dei diritti di ciascuno e nella consapevolezza delle loro responsabilità nei confronti delle generazioni future e della Terra, la grande avventura che fa di essa uno spazio privilegiato della speranza umana.

Si deduce, quindi, che il Diritto comunitario tratta l'Ambiente come cosa fondamentale alla Dignità della Persona Umana ed esige dagli Stati Europei che, effettivamente, imputino responsabilità a chi degrada l'Ambiente.

\section{Conclusioni}

Il Costituzionalismo contemporaneo ha eletto, tra gli oggetti del suo studio, il denominato Ambientalismo come strumento giuridico capace di propiziare la preservazione ambientale, come uno dei Diritti Umani più valorizzati nella Scienza del Diritto.

In effetti, l'Ambiente Ecologicamente Equilibrato è studiato come un Diritto Umano e, pertanto, intrinseco all'elenco dei Diritti Fondamentali.

Si corrobora la tesi difesa, ossia, l'inserzione della preservazione ambientale come diritto fondamentale dell'uomo dall'analisi dei testi delle costituzioni in tutto il mondo.

Si osserva nel Diritto Costituzionale Positivo elaborato negli ultimi cinquant'anni un'enorme preoccupazione del legislatore costituente nello stipolare che l'Ambiente è un valore giuridico inserito tra i Principi

47 NEVES, Marcelo. Transconstitucionalismo. São Paulo: M. Fontes, 2009. 
che guidano le Carte Magne e, solitamente, è disposto espressamente che si tratta di un Diritto Fondamentale.

Oltre all'Europa, culla della Scienza Giuridica, le costituzioni delle Americhe del Sud, del Nord e Centrale, l'Africa, l'Asia e l'Oceania hanno anche eletto l'Ambiente come qualcosa da essere protetta dagli Stati e dalla Società, costatandosi, dunque, che sia nel sistema Civil Law, sia nella Common Law le norme costituzionali e infra-costituzionali si sono, sempre di più, preoccupate dell'Ambiente.

Consapevole di tale imposizione costituzionale, il Diritto Comunitario, attraverso varie norme, soprattutto la Direttiva 2004/35, ha stabilito il sistema di Responsabilità per Danni all'Ambiente in Europa.

Nella riferita Direttiva, il Parlamento Europeo, in maniera encomiabile, ha stabilito direttrici che, con forza cogente, devono servire di guida alla costruzione delle legislazioni interne dei Paesi membri perché ci sia una corretta imputazione della Responsabilità Ambientale.

In effetti, come sostenuto dal testo, la semplice esistenza di norme di protezione dell'Ambiente poco contribuisce per una concreta inibizione del comportamento che degrada. In verità, la protezione ambientale sarà reale solo se accompagnata da un insieme di norme che diano Responsabilità Civile, Penale e Amministrativa a quelli che, deplorevolmente, distruggono l'Ambiente.

L'implacabile, rapida e giusta imputazione di Responsabilità a chi degrada, oltre al semplice aspetto riparatore e, eventualmente, punitivo, hanno una funzione pedagogica di estremo rilievo, nella misura i cui scoraggia nuovi comportamenti dannosi.

Si loda, pertanto, il contemporaneo trattamento che il Diritto Costituzionale conferisce all'Ambiente, eleggendolo comunemente come Principio Costituzionale e stabilendo regole d'imputazione a chi degrada, perché, tutti, universalmente, possano vivere in un Ambiente Ecologicamente Equilibrato e possano conferire alle future generazioni questa stessa benedizione.

\section{RIFERIMENTI}

ADEDE Y CASTRO, João Marcos. Resíduos perigosos no direito ambiental internacional: sua internalização nos países do Mercosul. Porto Alegre: Sergio A. Fabris Editor, 2003.
ÁFRICA DO SUL. Constituição África do Sul. Disponibile in: <http://www.juriscien cia.com/vademecum/ constituicoes-estrangeiras/constituicao-da-africa-dosul-constituti on-of-the-republic-of-south-africa/136>. Accesso in: 20 apr. 2016.

ALEMANHA. Lei Fundamental da República da Alemanba. Disponibile in: <https://www.btg-bestellservice.de/ pdf/80208000.pdf>. Accesso in: 20 apr. 2016.

ANGOLA. Constituição da República de Angola. Disponibile in: <http://www. governo.gov.ao/Arquivos/Constituicao_da_Republica_de_Angola.pdf $>$. Accesso in: 20 apr. 2016.

ARGENTINA. Código Civil de 2002. 2002. Disponibile in: <http://www.infoleg.gov.ar/ infolegInternet/ anexos/105000-109999/109481/texact.htm>. Accesso in: 14 mar. 2016.

ARGENTINA. Constituição Nacional de 1994. 1994. Disponibile in: <http://infoleg.mecon. gov.ar/infolegInternet/anexos/0-4999/804/norma.htm>. Accesso in: 14 mar. 2016.

ARGENTINA. Ley Nacional 25.675. Ley General del Ambiente. Disponibile in: <http://www2.medioambiente.gov.ar $/ \mathrm{mlegal} / \mathrm{marco} / \mathrm{ley} 25675 . \mathrm{htm}>$. Accesso in: 14 mar. 2016.

AUSTRÁlIA. Constituição da Austrália. Disponibile in: <http://www.egov.ufsc. br/portal/sites/default/files/ anexos/21382-21383-1-PB.htm>. Accesso in: 20 apr. 2016.

ÁUSTRIA. Bundesverfassungsgeset:. Disponibile in: <http://www.ris.bka.gv.at>. Accesso in: 20 apr. 2016.

BANERJEE, Debadyuti. Environmental Jurisprudence in India: a look at the initiatives of the supreme court of india and their success at meeting the needs of evirosocial justice. 2008. Disponibile in: <http://www. academia.edu/430162/EnvironmentaljurisprudenceinIndialookattheinitiativesoftheSupremeCourtofIndiaandtheir_success_at_meeting_the_needs_of_enviro-social_justice>. Accesso in 20 apr. 2016.

BEDRAN, K. M.; MAYER, E. A. Responsabilidade civil por danos ambientais no direito brasileiro e comparado: teoria do risco criado versus teoria do risco integral. Veredas do Direito, Belo Horizonte, v. 10, n. 19, jan./jul. 2013.

BÉLGICA. Constituição Federal. Disponibile in: <http://www.egov.ufsc.br/portal/ sites/default/files/ 
anexos/21393-21394-1-PB.htm>. Accesso in: 20 apr. 2016.

BOLÍVIA. Constituição Política do Estado Plurinacional da Bolivia. Disponibile in: <http://www.ftierra.org/index. $\mathrm{php} /$ generales/14-constitucion-politica-del-estado $>$. Accesso in: 05 apr. 2016.

BRASIL. Lei n.10.406, de 10 de janeiro de 2002. Institui o Código Civil. Diário Oficial da União, Brasília, 11 jan.2002. Disponibile in: <http:// www.planalto.gov. br/ccivil_03/leis/2002/110406.htm>. Accesso in: 13 apr. 2016.

BRASIL. Constituição (1988). Constituição da República Federativa do Brasil. Brasilia: Senado Federal, 1988. Disponibile in: <http://www.planalto.gov.br/ccivil03/ constituicao/constituicao.htm>. Accesso in: 13 apr. 2016.

BRASIL. Lei n. 6.938, de 31 de agosto de 1981. Dispõe sobre a Política Nacional do Meio Ambiente, seus fins e mecanismos de formulação e aplicação, e dá outras providências. Disponibile in: <http://www.planalto. gov.br/ccivil_03/leis/16938.htm>. Accesso in: 13 apr. 2016.

BULGÁRIA. Constituição. Disponibile in: < http://www. egov.ufsc.br/portal/sites/defa ult/files/anexos/2136221363-1-PB.htm>. Accesso in: 09 mag. 2016.

CANADÁ. Constituição. Disponibile in: <http://www. egov.ufsc.br/portal/sites/default / files/anexos/2136321364-1-PB.htm >. Accesso in: 09 mag. 2016.

CANOTILHO, José Joaquim Gomes. Direito constitucional e teoria da constituição. 4. ed. Coimbra: Almedina, 2000.

CHILE. Constituición Politica de La República de Chile. Disponibile in: <https:// www.camara.cl/camara/media/ docs/constitucionpolitica.pdf $>$. Accesso in: 13 apr. 2016.

CHINA. Constituição. Disponibile in: <http://bo.io.gov. mo/bo/i/1999/constituicao/ind ex.asp >. Accesso in: 13 apr. 2016.

CINGAPURA. Constituição. Disponibile in: <http:// www.egov.ufsc.br/portal/sites/de fault/files/ anexos/21400-21401-1-PB.html>. Accesso in: 13 apr. 2016.

COLÔMBIA Constituição. Disponibile in: <http:// www.jurisciencia.com/vademecum/ constituicoesestrangeiras/a-constituicao-da-colombia-constitucion- de-colombia/582/>. Accesso in 09 mag. 2016.

COLÔMBIA. Constituição. Disponibile in: <http:// www.jurisciencia.com/vademecum/ constituicoesestrangeiras/a-constituicao-da-colombia-constitucionde-colombia/582/>. Accesso in: 13 apr. 2016.

CUBA. Constituição. Disponibile in: < http://www.cuba. cu/gobierno/cuba.htm>. Accesso in: 09 mag. 2016.

EQUADOR. Constituição. Disponibile in: <http://www. stf.jus.br/repositorio/cms/ portalStfInternacional/ newsletterPortalInternacionalFoco/anexo/ConstituicaodoEquador.pdf Accesso in: 13 apr. 2016.

ESPANHA. Constituição da Espanha de 1978. 1978. Disponibile in: <https://www.iberred. org/pt/legislacion-penal/constituicao-espanhola-de-27-de-dezembro-de-1978>. Accesso in: 13 apr. 2016.

ESTADOS UNIDOS. Comprehensive environmental response, compensation, and liability act. Disponibile in: <http:// www.epa.gov/superfund/policy/index.htm>. Accesso in: 28 apr. 2016.

EUROPA. Constituição. Disponibile in: <http://eurlex.europa.eu/legal-content/IT/TXT/?uriCELEX\% 3A52003XX0718(01)>. Accesso in: 28 apr. 2016.

EUROPA. Diretiva da responsabilidade ambiental 2004/35 do parlamento europeu. Disponibile in: < http://ec.europa. eu/environment/legal/liability/pdf/eldbrochure/ ELD\%20brochure.pdf>. Accesso in: 21 apr. 2016.

FRANÇA. Carta Ambiental. 2004. Disponibile in: $<$ http://www.conseil-constitutionnel.fr/conseilconstitutionnel/root/bankmm/portugais/constitutionportugais.pdf>. Accesso in: 21 apr. 2016.

FRANÇA. Constituição. 1958. Disponibile in: <http:// www.conseil-constitutionnel.fr/conseilconstitutionnel/ root/bank_mm/portugais/constitution_portugais. pdf>. Accesso in 21 apr. 2016.

GIANNINI, Daniele. La tutela dell'ambiente. Disponibile in: <https://www.iusexplorer.it/Publica/FascicoloDossier/Latuteladellambiente/?idDocMaster40659 82\&idDataBanks19\&canale13\#>. Accesso in $06 \mathrm{mag}$. 2016.

GUSMAI, Antonio. La tutela costituzionale dell'ambiente tra valori (meta-positivi), interessi (mercificatori) e (assenza di) principi fondamentali. Disponibile in: <http:// www. edizioniesi.it/dperonline/data/uploads/articoli/tutelagusmai.pdf>. Accesso in: 06 mag. 2016. 
HOLANDA. Constituição Holandesa. Disponibile in: $<$ http:/ /www.oefre.unibe.ch/law /icl/nl00000_. html>. Accesso in: 09 mag. 2016.

IHERING, Rudolf Von. El fin en el derecho. Madrid: B. Rodriguez Serra, 1911.

INDIA. Constituição da República. 1950. Disponibile in: <http://lawmin.in/olwing/ coi/coi-english/coi-indexenglish.htm>. Accesso in: 20 apr. 2016.

INDIA. The environment protection act. 1986. Disponibile in: <http://envfor.nic. in/legis/env/env1.html>. Accesso in: 20 apr. 2016.

ITALIA. Constiturione dela Repubblica Italiana. Disponibile in: <http://www. quirinale.it/qrnw/statico/costituzione/pdf/Costituzione.pdf> . Accesso in: 20 apr. 2016.

JAPÃO. Constituição. Disponibile in: <http://www. br.emb-japan.go.jp/cultura/pdf/ constituicao.pdf $>$. Accesso in: 20 apr. 2016.

JAPÃO. Embaixada. Avanços foram feitos, mas há novos desafios a serem enfrentados. Disponibile in: <http://www. br.emb-japan.go.jp/cultura/ambiente.html>.Accesso in: 06 mag. 2016.

LAWYERS, Blakes. Environment law in Canada. 2012. Disponibile in: <http://www.blakesfiles.com/Guides/2012_Blakes_Environmental_Law_in_Canada_ EN.pdf>. Accesso in: 20 apr. 2016.

LIMARDI, Gianluca; SACCO, Sonia. Danno ambientale e rischio inquinamento: responsabilità civile e aspetti gestionali: inquadramento normativo del danno ambientale e rivolti nell'ambito della responsabilità civile. Disponibile in: <http://www.studiolimardi.it/files/lucca_2011. pdf $>$. Accesso in: 25 apr. 2016.

MÉXICO. Constitución Politica de los Estados Unidos Mexicanos. Disponibile in: <http://www.constitucion.gob. $\mathrm{mx} />$. Accesso in 21 apr. 2016.

MOÇAMBIQUE: Constituição. Disponibile in: < http:// www.presidencia.gov.mz/files / republica/constituicao_republica_moc.pdf $>$. Accesso in: 21 apr. 2016.

MOTOKI, Masato. Observations on environmental ethics, animal rights and culture. In: ASIAPACIFIC perspectives on environmental ethics. Bangkok: UNESCO Bangkok, 2008.

NEVES, Marcelo. Transconstitucionalismo. São Paulo: M. Fontes, 2009.
NORUEGA. Constituição da Noruega. Disponibile in: <https://pt.glosbe.com/pt/en/ Constitui $\%$ C3\%A7\%C3\%A30\%20da $\% 20$ Noruega $>$. Accesso in: 13 apr. 2016.

NORUEGA. Pollution control act. Disponibile in <https://www.regjeringen.no/ en/dokumenter/pollution-control-act/id171893/>. Accesso in: 21 apr. 2016.

PERU. Constitución Política Del Perú de 1993. Disponibile in: <http://vlex.com/vid/ constitucion-politica.peru42814763>. Accesso in: 13 apr. 2016.

PORTUGAL. Constituição (1976). Constituição da República Portuguesa. 1976. Disponibile in: <http://www. parlamento.pt/Legislacao/Paginas/ConstituicaoRepublica Portuguesa.aspx>. Accesso in: 20 apr. 2016.

PORTUGAL. Lei n. 11 de 7 de abril de 1987. Lei de Bases do Ambiente. Diário da República, n. 81, 1987. Disponibile in: <http://dre.pt/ pdf1sdip/1987/04/08100/13861397.pdf >. Accesso in: 23 de setembro 2016.

RODOLFI, Marco. Il dannoambientale e lerecentimodifiche legislative. 2013. Disponibile in: http://www.anra.it/Content/rishi.aspx?id=37 Accesso in: 25 apr.2016.

SENATORE, Gianluca. Lo sviluppo sostenibile. Profili giuridico-filosofici. Edizioni Nuova Cultura, 2008.

SOUZA, Mauro César Martins de; YAMAGUCHI, Taylla Evellyn. França: a construção do direito ambiental em um país desenvolvido. TÓPOS, v. 5, n. 2, p. 47-66, 2011. Accesso in: <http://revista.fct.unesp.br/index. $\mathrm{php} /$ topos/article/viewFile/2284/2089>. Accesso in: 24 apr. 2016.

SUÍÇA. Bundesverfassung der Schweizerischen Eidgenossenschaft. Disponibile in: <http://www.admin.ch>. Accesso in: 20 apr. 2016.

VENEZUELA. Constitución de la República Bolivariana de Venezuela. Asamblea Nacional Constituyente. Caracas, 1999 Disponibile in: <http://www.tsj.gov.ve/legislacion/constitucion1999.htm $>$. Accesso in :15 maio 2016

VOLLERO. Flora. Diritti umani e diritti fondamentali fra tutela costituzionale e tutela sovranazionale: il diritto ad un ambiente salubre. Disponibile in <http:/ / files.studiperlapace.it/spp_zfiles/docs/20041205175248.pdf $>$. Accesso in: 06 maio 2016. 
Para publicar na Revista de Direito Internacional, acesse o endereço eletrônico www.rdi.uniceub.br ou www.brazilianjournal.org.

Observe as normas de publicação, para facilitar e agilizar o trabalho de edição. 